\title{
Symmetry considerations in the empirical k.p Hamiltonian for the study of intermediate band solar cells
}

\author{
A. Luque , A. Mellor, E. Antolín, P.G. Linares, I. Ramiro, I. Tobías, A. Martí
}

\begin{abstract}
A B S T R A C T
With the purpose of assessing the absorption coefficients of quantum dot solar cells, symmetry considerations are introduced into a Hamiltonian whose eigenvalues are empirical. In this way, the proper transformation from the Hamiltonian's diagonalized form to the form that relates it with $\Gamma$ point exact solutions through k.p envelope functions is built accounting for symmetry. Forbidden transitions are thus determined reducing the calculation burden and permitting a thoughtful discussion of the possible options for this transformation. The agreement of this model with the measured external quantum efficiency of a prototype solar cell is found to be excellent.
\end{abstract}

\section{Introduction}

The use of nanostructures in solar cells has stirred considerable interest because of potential improvements. In particular, quantum dots (QDs) have been proposed to form intermediate bands or levels within the barrier material bandgap $[1,2]$ and thus yield subbandgap current while keeping the voltage unchanged $[3,4]$. The efficiency limit for this concept is $63 \%$ vs. $41 \%$ for ordinary solar cells (blackbody spectrum, 6000/300 K for Sun/cell temperature) [5].

Intermediate band solar cells (IBSCs) along the lines mentioned have been manufactured by several groups [6-9] by forming InAs QDs within a GaAs bulk material (or barrier material). Best efficiency is $18.32 \%$ [10]. IBSCs have already known an incipient industrial use in triple junction solar cells [11]. Unfortunately, the generation of subbandgap current has been in all cases too small. For any improvement, understanding the nature of the optical transitions associated to the generation of electron hole pairs is a necessity; this is impossible without a quantum picture of the mechanisms involved.

The introduction of the QDs into the bulk semiconductor induces the appearance of localized states detached from the conduction band (CB) and the valence band (VB) with energies that are within the bulk semiconductor bandgap. In addition, the QDs modify the extended states with energies within the bands of the bulk semiconductor. In the IBSC formed with QDs, the IB is the lowest-energy confined-state detached from the $\mathrm{CB}$. The transitions from this state to the rest of the $\mathrm{CB}$ states - bounded or extended - are one of the aspects to be understood. A single band effective mass treatment is enough for this task and a simplified model has already been published on this topic, involving the energy spectrum of the $C B$ detached states as well as the transition between confined states [12,13] and between these and the extended states modified by the QD presence [14].

However, for confined states detached from the VB and for the transitions between these states and the IB or the other confined states detached from the $\mathrm{CB}$, the single band effective mass treatment is not adequate. The treatment has to consider simultaneously the $\mathrm{CB}$ and the different bands composing the VB: the light-hole $(l h)$, the heavy-hole $(h h)$ and the split-off (so) bands. Within the effective mass framework [15] (Ref. Ch. 6), the simplest configuration to explain adequately the transitions between these bands is a four band k - p model. A study of the transitions from the different $V B s$ and the $C B$ that reproduced rather accurately the measured quantum efficiency was published recently [16] using an early form of this model. The purpose of this paper is to refine the model taking into account symmetry considerations.

There are many motives for introducing symmetry considerations into the aforementioned model. First, symmetry is a very fundamental property of nature and should be considered in theoretical studies. Second, there are uncertainties about the form of the approximations used in the said model that can be reduced by the use of symmetry properties. In this paper, these uncertainties 
are clearly stated. It is found that the choice of a specific form of the model among the several that are possible has little effect on the final result, which is in good agreement with experimental data. Finally, symmetry considerations greatly reduce the calculation time following the trend in quantum calculations.

This paper is organized as follows: In Section 2 we introduce the $\mathrm{k}$ - p method and the representation theory applied to the case in study; we also derive an important relationship that relates the $\mathbf{r}$ and r symmetries. In Section 3 we discuss the modifications in the Hamiltonian presented previously [16] taking into account symmetry considerations. In particular we discuss the transformation matrix $\left\{T_{\alpha}\right\}$ that diagonalizes this Hamiltonian, we discuss the symmetry properties of the eigenfunctions of this Hamiltonian and we explain how to derive the envelope functions. In Section 4 we describe the optical matrix elements and we apply symmetry considerations to determine the forbidden transitions. In Section 5 we apply the matrix elements to obtain absorption coefficients, calculate quantum efficiency and compare it with experimental data and prior calculations [16]. Finally, in Section 6, we draw some conclusions.

\section{Theoretical background}

\subsection{The k.p method}

The k.p model is widely used in the study of solids [15,17], being especially well suited to nanostructured solids. The wavefunctions are expanded in a basis of orthonormal functions which are labeled by two indices - the band index $v$ and the wavevector k. For each band, the eigenfunction takes the form of a plane wave multiplied by $u_{0, v}$, which is the exact band-specific eigenfunction of the Hamiltonian evaluated at the $\Gamma$-point of reciprocal space $(\mathbf{k}=0)$. In this way the wavefunctions are

$$
\begin{aligned}
& \Xi(\mathbf{r})=\sum_{v} u_{0, v}(\mathbf{r}) \Psi_{v}(\mathbf{r}) \\
& \Psi_{v}(\mathbf{r})=\frac{1}{\sqrt{\Omega}} \sum_{\mathbf{k}} \psi_{v}(\mathbf{k}) e^{i \mathbf{k} \cdot \mathbf{r}}
\end{aligned}
$$

where $\Omega$ is the volume occupied by the wavefunctions; usually this is a cube of side $L$ so that $\Omega=L^{3}$. Furthermore, in order to respect periodic conditions at the edges of this cube, $\mathbf{k}=2 \pi \mathbf{\kappa} / L, \mathbf{\kappa}$ being triplets of integers. $\psi_{v}(\mathbf{k})$ are coefficients that determine the wavefunction $\Xi(\mathbf{r})$.

The $u_{0, v}$ functions, that we call involutes, present variations on the scale of the atoms in the crystalline lattice and follow their symmetry and periodicity. On the contrary, the functions $\Psi_{v}(\mathbf{r})$, called envelopes, are affected by the size and symmetry of the nanostructure. In the case of zincblend crystals, the orbital part of the involute wavefunctions is represented by $|X\rangle,|Y\rangle,|Z\rangle,|S\rangle$ (the $X-, Y-, Z$ and $S$-involutes) with the symmetry of $x\left(x^{2}+y^{2}+z^{2}\right), y\left(x^{2}+y^{2}+z^{2}\right)$, $z\left(x^{2}+y^{2}+z^{2}\right)$ and $\left(x^{2}+y^{2}+z^{2}\right)$, respectively [15]. In the four band model, the spin coordinates are neglected. In other words, each orbital wavefunction is doubly degenerate with spins $1 / 2$ or $-1 / 2$. We adopt this simplification because we are looking for the simplest Hamiltonian able to construe the semiconductor energy bands.
The envelope functions that multiply the $X-, Y$-, $Z$ - and $S$-involutes are called the $X-, Y_{-}, Z-$, and $S$-envelopes.

\subsection{The $Q D$ symmetry}

InAs QDs in GaAs form strongly truncated quadrangular pyramids. They induce a change of the material CB and VB. In our simplifying effort, we assume the QD to be a squat parallelepiped and adopt a mesoscopic potential box of parallelepipedic shape.

A parallelepipedic box has the $\mathrm{D}_{4 \mathrm{~h}}$ symmetry group that includes the identity $E$, 4-fold vertical rotations $\left(C_{4},-C_{4}, C_{2}\right)$ two 2 -fold rotations $C^{\prime}$ around the base diagonals, two 2 -fold rotations $C^{\prime \prime}$ around the $x$ and $y$ axes and the product of the preceding transformations times the center inversion $I$. In total this group has 16 symmetry operations. Table 1 shows all the cited operations and how the vector $(x, y, z)$ is transformed by them.

Any function, no matter how complicated it might be, can generate a vector space in the following way: the function is modified by the symmetry operations and the new functions generated are the basis of such a space, whose dimension is, in the worst case, the number $N_{G}$ of operations of the group (16 in this case). However, for certain functions, many of the transformed functions are e.g., the original function (or linear combinations of some few transformed functions) so leading to spaces of lower dimension.

Each symmetry operation $G$ of the group $\boldsymbol{G}$ induces a transformation in the vector space that is represented by a matrix $\Gamma_{G}$. These matrices constitute a representation of the group. If all the transformed functions are linearly independent, the dimension of the matrices is $N_{G}$ and the representation is called the regular representation. If the function generating the representation is highly symmetric, the dimension of the space generated may be very small, even one. In the latter case, the function is invariant under the group operations with all matrices of dimension one with a single matrix element of value 1 (the identical representation) or at most (if the function is real) \pm 1 .

Sometimes, combination of the basis functions leads to matrices that are diagonal by blocks, each block corresponding to smaller dimension representations. Actually, in finite groups, all the representations are unitary [18] (Vol. 2 page 945). This means that the matrices are unitary (the lines and columns are orthonormal vectors) and a basis, formed of linear combinations of the initial basis, can be found in which the representation matrices are diagonal in blocks. Such matrices are called the direct sum of the diagonal blocks. Additionally, a basis can be found where these diagonal blocks cannot be further reduced: they are called irreducible representations [18-21]. We say that the representation $\boldsymbol{\Gamma}$ has been developed into the direct sum of irreducible representations $\Gamma^{(i)}$; that is, $\Gamma_{G}=\sum_{i} \Gamma_{G}^{(i)}$.

The traces of the representation matrices $\chi_{i}$ are called the characters of the representation. The table of characters of the irreducible representations of the $\mathrm{D}_{4 \mathrm{~h}}$-symmetry group, as taken from the literature $[19,20]$, is presented in Table 2 . In the table, the irreducible representations and the symmetry operations grouped in classes (whose exact meaning is not relevant here) appear. In the rightmost column, some basis functions generating

Table 1

\begin{tabular}{|c|c|c|c|c|c|c|c|}
\hline$E$ & $I C^{\prime \prime}{ }^{\prime}$ & $I C^{\prime \prime}{ }_{y 2}$ & $I C_{2}$ & $I$ & $C^{\prime \prime}{ }_{x 2}$ & $C^{\prime \prime}{ }_{y 2}$ & $c_{2}$ \\
\hline$+x,+y,+z$ & $-x,+y,+z$ & $+x,-y,+z$ & $+x,+y,-z$ & $-x,-y,-z$ & $+x,-y,-z$ & $-x,+y,-z$ & $-x,-y,+z$ \\
\hline$I C_{d s 2}^{\prime}$ & $-C_{4}$ & $C_{4}$ & $C_{d p 2}$ & $C_{d s 2}^{\prime}$ & $I\left(-C_{4}\right)$ & $I C_{4}$ & $I C_{d p 2}^{\prime}$ \\
\hline$+y,+x,+z$ & $-y,+x,+z$ & $+y,-x,+z$ & $+y,+x,-z$ & $-y,-x,-z$ & $+y,-x,-z$ & $-y,+x,-z$ & $-y,-x,+z$ \\
\hline
\end{tabular}

Symmetry operations of the $\mathrm{D}_{4 h}$ group. 
Table 2

Table of characters of the $\mathrm{D}_{4 \mathrm{~h}}$ group of symmetry.

\begin{tabular}{llrrrrrrrrrl}
\hline Ch.D $4 \mathrm{~h}$ & $E$ & $C_{2}$ & $2 C_{4}$ & $2 C_{2}$ & $2 C_{2}^{\prime \prime}$ & $I$ & $I C_{2}$ & $2 I C_{4}$ & $2 I C_{2}$ & $2 I C^{\prime \prime}$ & \\
\hline$A_{1 g}$ & 1 & 1 & 1 & 1 & 1 & 1 & 1 & 1 & 1 & 1 & $x^{2}+y^{2}, z^{2}$ \\
$A_{1 u}$ & 1 & 1 & 1 & 1 & 1 & -1 & -1 & -1 & -1 & -1 & \\
$A_{2 g}$ & 1 & 1 & 1 & -1 & -1 & 1 & 1 & 1 & -1 & -1 & \\
$A_{2 u}$ & 1 & 1 & 1 & -1 & -1 & -1 & -1 & -1 & 1 & 1 & $z$ \\
$B_{1 g}$ & 1 & 1 & -1 & 1 & -1 & 1 & 1 & -1 & 1 & -1 & $x^{2}-y^{2}$ \\
$B_{1 u}$ & 1 & 1 & -1 & 1 & -1 & -1 & -1 & 1 & -1 & 1 & \\
$B_{2 g}$ & 1 & 1 & -1 & -1 & 1 & 1 & 1 & -1 & -1 & 1 & $x y$ \\
$B_{2 u}$ & 1 & 1 & -1 & -1 & 1 & -1 & -1 & 1 & 1 & -1 & \\
$E_{g}$ & 2 & -2 & 0 & 0 & 0 & 2 & -2 & 0 & 0 & 0 & $(x z, y z)$ \\
$E_{u}$ & 2 & -2 & 0 & 0 & 0 & -2 & 2 & 0 & 0 & 0 & $(x, y)$ \\
\hline & & & & & & & & & &
\end{tabular}

irreducible representations that are frequently found in practical applications are listed according to their irreducible representation.

Note that the lines are orthogonal vectors of dimension 16 if the classes are split into the symmetry operations they are formed of (e.g., $2 C^{\prime \prime}$ is split into $C^{\prime \prime}{ }_{x 2}$ and $C^{\prime \prime}{ }_{y 2}$, both with the same character that depends on the representation). The norm of these vectors squared is $N_{G}(=16)$. This is the test to know if a representation is irreducible.

Once the characters $\chi_{G}$ of a given representation are known, its development as the direct sum of irreducible representations can involve the repetition of $h^{(i)}$ times each irreducible representation. The well known formula for the $h^{(i)}$ coefficients is [19] (page 454)

$h^{(i)}=\sum_{G=1}^{N_{G}} \chi_{G} \chi_{G}^{(i) *} / N_{G}$

where the index (i) refers to the irreducible representation and the index $G$ corresponds to the group operation, (note: the classes must be split into their symmetry operations). The asterisk represents complex conjugation (unnecessary for $\mathrm{D}_{4 \mathrm{~h}}$ ).

Notice that the bases we are referring to in this subsection involve totally different vector spaces than those in Section 2.1. The coexistence of Hilbert vector space associated to the Hamiltonian and of finite dimension vector spaces associated to the representation of the $\mathrm{D}_{4 \mathrm{~h}}$ group symmetry operations will persist throughout the paper. We shall try to keep clear the difference.

\subsection{Relating the $r$ and $k$ point-symmetries}

In the k.p method, the envelope functions result from a plane wave development as indicated in Eq. (1), second line. In practice, the assumption of a finite number of plane waves results in the determination of the wavefunction at a finite number of nodes of a calculation cubic lattice. The rest of the points, if needed, are to be interpolated. Mathematically speaking, the coefficients $\psi_{v}(2 \pi \mathbf{\kappa} / L)$ of the development in Eq. (1), that we shall also call $\psi_{y, k}$, are the Discrete Fourier Transform (DFT) of the values of $\Delta \Omega^{1 / 2} \Psi_{v}(\mathbf{r})$ calculated at the lattice nodes where $\Delta \Omega=(L / N)^{3}=l^{3}$ is the element of volume associated to a calculation node and, obviously, $\Omega=\Delta \Omega \times N^{3}$. The values of $\Delta \Omega^{1 / 2} \Psi_{v}(\mathbf{n} L / N)$ at these nodes ( $\boldsymbol{n}$ like $\boldsymbol{\kappa}$ are triplets of integers) are called $\Psi_{v, \mathbf{n}}$ and they are the inverse transform (IDFT) of $\psi_{v, \boldsymbol{\kappa}}$. Sometimes, the sub-index $v$ is dropped for simplicity. Therefore, with the simplified notation, the DFT and the IDFT are defined, respectively as

$$
\begin{gathered}
\psi_{\mathbf{k}}=\frac{1}{N^{3 / 2}} \begin{array}{c}
(N-1) / 2,(N-1) / 2,(N-1) / 2 \\
n_{x}=-(N-1) / 2, n_{y}=-(N-1) / 2, n_{z}=-(N-1) / 2 \\
(N-1) / 2,(N-1) / 2,(N-1) / 2 \\
\Psi_{\mathbf{n}}=\frac{1}{N^{3 / 2}} \psi_{k_{x}=-(N-1) / 2, k_{y}}=-(N-1) / 2, k_{z}=-(N x-1) / 2
\end{array} \\
\psi_{\mathbf{k}} e^{2 \pi i \mathbf{k} \cdot \mathbf{n} / N}
\end{gathered}
$$

The sum terms depend on three integer indices as explained in the text. This is perhaps not the most common way of defining the DFT and the IDFT. It is more frequent to use natural numbers (not negative integers) for the indices starting with the term of zero exponent. Here the zero exponent is to be found in the middle of the series for each index. The usual definition can be changed into the one used here if, before the transformation, the array of numbers suffers a circular permutation of $(N-1) / 2$ positions to the left with respect to each index, the ordinary DFT is obtained and the resulting array of numbers is again rotated $(N-1) / 2$ times to the right for each index.

Let $G$ be a symmetry operation belonging to the group $G$ that is a subgroup of the cube symmetry $\mathrm{O}_{\mathrm{h}}$ (as the $\mathrm{D}_{4 \mathrm{~h}}$ symmetry is). By definition,

$G \Psi_{\mathbf{n}}=\Psi_{G^{-1} \mathbf{n}}$

It can be proven that [21] (Ref. pag. 107)

$G \psi_{\kappa}=\psi_{G^{-1}}$ K

Let us now assume that $\Psi_{\mathbf{n}}$ is transformed by the symmetry operations of $\boldsymbol{G}$ according to the representation matrices $\Gamma_{G}$ (it could be one of the irreducible representations in Table 2). Let $\left\{\Psi_{\mathrm{n}}^{i}\right\}$ be the set of basis functions of this representation. Then,

$G \Psi_{\mathbf{n}}^{i}=\Psi_{G^{-1} \mathbf{n}}^{i}=\sum_{j} \Gamma_{G}^{i, j} \Psi_{\mathbf{n}}^{j}$

The DFT of $G \Psi_{\mathbf{n}}^{i}$ is, by definition

$$
\begin{aligned}
G \psi_{\mathbf{K}}^{i}= & \frac{1}{N^{3 / 2}} \sum_{\mathbf{n}} G \Psi_{\mathbf{n}}^{i} e^{-2 \pi i \boldsymbol{\kappa} \cdot \mathbf{n} / N} \\
& =\frac{1}{N^{3 / 2}} \sum_{\mathbf{n}} \sum_{j} \Gamma_{G}^{i, j} \Psi_{\mathbf{n}}^{j} e^{-2 \pi i \mathbf{k} \cdot \mathbf{n} / N}=\sum_{j} \Gamma_{G}^{i, j} \psi_{\mathbf{\kappa}}^{j}=\psi_{G^{-1} \mathbf{\kappa}}
\end{aligned}
$$

hence the DFT and the IFDT are transformed by the symmetry operations according to the same representation and therefore they have the same table of characters (note that the basis index and the imaginary unity are both represented by $i$ ).

\section{The empiric k.p Hamiltonian (EKPH)}

Neglecting for the moment the spin orbit coupling and the lattice strain, the development of the Hamiltonian in the (Hilbert space) basis indicated in Eq. (1) takes a rather simple form [15,16] that is presented in Table 3 (upper part). Note that only the terms involving two equal $\boldsymbol{k}$ vectors are non zero and hence we obtain, in this four band $\mathrm{k} \cdot \mathrm{p}$ approximation, a four dimensional matrix $\{H\}$ whose terms are functions of $\left(k_{x}, k_{y}, k_{z}\right)$.

The eigenvalues, also represented in the same table ("Eigenvalues" column), show four values (each one with two spin states) for every $\boldsymbol{K}$ ( $\boldsymbol{k}$ normalized to $1 / d$ ), corresponding (from top to bottom) to the CB, the light hole VB and a doubly degenerate VB state. The doubly degenerate $\mathrm{VB}$ eigenvalues present a positive effective mass that is known to be impossible (it would fill the band gap). Thus, the assumption of no spin-orbit interaction and neglecting the effect of the lattice strain is not acceptable within the four band k.p model.

The eigenvectors corresponding to the mentioned (non-acceptable) eigenvalues are also represented in Table 3 in the column "Eigenvectors" (each eigenvector is a given as a row adjacent to the corresponding eigenvalue). The doubly degenerate eigenvalue defines a two-dimensional space of vectors all of which are normal to the other two one-dimensional eigenvectors. In this two-dimensional space, the choice of the basis is arbitrary. We have taken an orthogonal basis formed of two vectors all of whose elements transform in the $\mathrm{D}_{4 \mathrm{~h}}$ group operations according to irreducible representations (this was not the case in Ref. [16]). In effect (Table 2), the eigenvector components at the top of the "Eigenvectors" column transform as the irreducible representations $\left\{E_{u}, E_{u}\right.$, $\left.A_{2 u}, A_{1 g}\right\}$; the next eigenvector below transforms in the same way, the following one as $\left\{E_{u}, E_{u}, 0,0\right\}$, and the one at the bottom as $\left\{E_{g}\right.$, 
Table 3

Hamiltonian matrix, eigenvalues and eigenvectors when the spin-orbit coupling is neglected and proposed eigenvalues.

\begin{tabular}{|c|c|c|c|c|c|}
\hline$H_{\nu, v^{\prime}}(\mathbf{k})=\frac{1}{\Omega_{\text {cell }}} \int u_{\nu, 0}^{*} e^{-i \mathbf{k} \cdot \mathbf{r}} H u_{v^{\prime}, 0} e^{i \mathbf{k} \cdot \mathbf{r}} d^{3}$ & $|X\rangle$ & $|Y\rangle$ & $|Z\rangle$ & $|S\rangle$ & \\
\hline$\langle X|$ & $A K$ & 0 & 0 & $B K_{x}$ & \\
\hline$\langle Y|$ & 0 & $A K$ & 0 & $B K_{y}$ & \\
\hline$\langle Z|$ & 0 & 0 & $A K$ & $B K_{z}$ & \\
\hline$\langle S|$ & $B K_{x}$ & $B K_{y}$ & $B K_{z}$ & $E_{g}+A K$ & \\
\hline Eigenvalues & Eiger & & & & Proposed eigenvalues \\
\hline$E_{c b}(K)=E_{g} / 2+A K^{2}+\sqrt{\left(E_{g} / 2\right)^{2}+B^{2} K^{2}}$ & $K_{x}$ & $K_{y}$ & $K_{z}$ & $\frac{E_{g}^{2}+\sqrt{E_{g}^{2}+4 B^{2} K^{2}}}{2 B}$ & $E_{c b}(K) \cong E_{g}+\frac{h^{2} K^{2}}{2 m_{c b} d^{2}}$ \\
\hline$E_{i h}(K)=E_{g} / 2+A K^{2}-\sqrt{\left(E_{g} / 2\right)^{2}+B^{2} K^{2}}$ & $K_{x}$ & $K_{y}$ & $K_{z}$ & $\frac{E_{g}^{2}-\sqrt{E_{g}^{2}+4 B^{2} K^{2}}}{2 B}$ & $E_{l h}(K) \cong-\frac{\hbar^{2} K^{2}}{4 m_{l f} d^{2}}$ \\
\hline$E_{\nu 2}(K)=A K^{2}$ & $-K_{y}$ & $K_{x}$ & 0 & 0 & $E_{h \hbar}(K) \cong-\frac{\hbar^{2} K^{2}}{2 m_{h \hbar} d^{2}}$ \\
\hline $\mathrm{E}_{\mathrm{v} 1}(K)=A K^{2}$ & $K_{x} K_{z}$ & $K_{y} K_{z}$ & $-\left(K_{x}^{2}+K_{y}^{2}\right)$ & 0 & $E_{s 0}(K) \cong-E_{s}-\frac{\hbar^{2} K^{2}}{2 m_{s 0} d^{2}}$ \\
\hline
\end{tabular}

Table 4

Summand matrices $\left\{T_{\alpha A}\right\}$ and $\left\{T_{\alpha B}\right\}$ into which the rotated transformation matrix $\left\{T_{\alpha}\right\}$ is decomposed.

$$
\begin{aligned}
& \left\{T_{\alpha A}\right\}=\left(\begin{array}{llll}
K_{x} / N_{1} & K_{y} / N_{1} & K_{z} \sqrt{1-\alpha^{2}} / N_{1} & \left(E_{G}+\sqrt{E_{G}^{2}+4 B^{2}\left(K_{x}^{2}+K_{y}^{2}+K_{z}^{2}\right)}\right) \sqrt{1-\alpha^{2}} / 2 B N_{1} \\
K_{x} / N_{2} & K_{y} / N_{2} & K_{z} \sqrt{1-\alpha^{2}} / N_{2} & \left(E_{G}-\sqrt{E_{G}^{2}+4 B^{2}\left(K_{x}^{2}+K_{y}^{2}+K_{z}^{2}\right)}\right) \sqrt{1-\alpha^{2}} / 2 B N_{2} \\
-K_{y} \sqrt{1-\alpha^{2}} / N_{3} & K_{x} \sqrt{1-\alpha^{2}} / N_{3} & 0 & 0 \\
K_{x} K_{z} \sqrt{1-\alpha^{2}} / N_{4} & K_{y} K_{z} \sqrt{1-\alpha^{2}} / N_{4} & -\left(K_{x}^{2}+K_{y}^{2}\right)\left(1-\alpha^{2}\right) / N_{4} & 0
\end{array}\right) \\
& \left\{T_{\alpha B}\right\}=\alpha\left(\begin{array}{cccc}
0 & 0 & \left(E_{G}+\sqrt{E_{G}^{2}+4 B^{2}\left(K_{x}^{2}+K_{y}^{2}+K_{z}^{2}\right)}\right) / 2 B N_{1} & -K_{z} / N_{1} \\
0 & 0 & \left(E_{G}-\sqrt{E_{G}^{2}+4 B^{2}\left(K_{x}^{2}+K_{y}^{2}+K_{z}^{2}\right)}\right) / 2 B N_{2} & -K_{z} / N_{2} \\
K_{x} K_{z} / N_{4} & K_{y} K_{z} / N_{4} & -\left(K_{x}^{2}+K_{y}^{2}\right)\left(1-\alpha^{2}\right) / N_{4} & \left(K_{x}^{2}+K_{y}^{2}\right) \alpha / N_{4} \\
K_{y} / N_{3} & -K_{x} / N_{3} & 0 & -\left(K_{x}^{2}+K_{y}^{2}\right) \sqrt{1-\alpha^{2}} / N_{3}
\end{array}\right) \\
& N_{1}=\sqrt{8 B^{2}\left(K_{x}^{2}+K_{y}^{2}+K_{z}^{2}\right)+2 E_{g}\left(E_{g}+\sqrt{E_{g}^{2}+4 B^{2}\left(K_{x}^{2}+K_{y}^{2}+K_{z}^{2}\right)}\right)} / 2 B \\
& N_{2}=\sqrt{8 B^{2}\left(K_{x}^{2}+K_{y}^{2}+K_{z}^{2}\right)+2 E_{g}\left(E_{g}-\sqrt{E_{\mathrm{g}}^{2}+4 B^{2}\left(K_{x}^{2}+K_{y}^{2}+K_{z}^{2}\right)}\right)} / 2 B \\
& N_{3}=\sqrt{\left(K_{x}^{2}+K_{y}^{2}\right)} ; \quad N_{4}=\sqrt{\left(K_{x}^{2}+K_{y}^{2}\right)\left(K_{x}^{2}+K_{y}^{2}+k_{z}^{2}\right)} ;
\end{aligned}
$$

$\left.E_{g}, A_{1 g}, 0\right\}$. Zero, like any constant, transforms according to the identical representation $A_{1 g}$, but by putting 0 we express that when it appears (e.g., in a product) it leads to a zero value, beyond any symmetry consideration.

Notice that the four eigenvectors are mutually orthogonal. Each eigenvector, once normalized, forms a row of a unitary matrix $\{T\}$ such that $\left\{H_{d}\right\}=\{T\}\{H\}\left\{T^{+}\right.$\} forms a diagonal matrix whose elements are the eigenvalues of $\{H\}$, the latter being the fourdimensional Hamiltonian matrix in the upper part of Table 3.

It is to be noticed that the normalization process does not affect the symmetry of each element. In fact, the norm of any of the eigenvectors follows the identical representation $\left(A_{1 g}\right)$ and therefore transforms unchanged in any symmetry operation.

One basic assumption of the EKPH approximation is that the eigenvalues (column "Proposed Eigenvalues") follow parabolic shapes with the experimental effective masses. The fact that the effective masses are positive for the conduction band and negative for the valence band makes experimentally consistent the new Hamiltonian that these eigenvalues are creating. With these eigenvalues we can build a new diagonal Hamiltonian $\left\{H_{d}^{\prime}\right\}$ that takes implicitly into account the spin orbit coupling and the crystal strain. Furthermore, the fact that the bands are taken to be parabolic erases any memory of the crystalline structure of the materials involved so that the only relevant symmetry is the $D_{4 h}$ of the nanostructure (although the involutes keep their own symmetry). This diagonal Hamiltonian is enough to obtain the eigenvalues of the nanostructure as we shall see in Section 3.1. It is considered to be rather accurate.

A second basic assumption of the EKPH is the choice of the transformation matrix that restores the Hamiltonian matrix to the initial basis functions expressed in the definition of the matrix elements appearing in Table 3 (left upper corner). We assume, in a first instance, that this matrix is the matrix $\{T\}$ already defined so that $\left\{H^{\prime}\right\}=\left\{T^{+}\right\}\left\{H_{d}^{\prime}\right\}\{T\}$. This is the same assumption taken in Ref. [16], but here $\{T\}$ has taken into account the $D_{4 h}$ symmetry, as explained above. However, it is to be understood that the choice made is rather arbitrary and therefore we propose now a generalization that embraces any possible choice of the basis vector in the subspace of the $\{H\}$ degenerate eigenvalue.

For this, we apply to $\{T\}$ the unitary transformation:

$\{R\}=\left(\begin{array}{cccc}1 & 0 & 0 & 0 \\ 0 & 1 & 0 & 0 \\ 0 & 0 & \sqrt{1-\alpha^{2}} & \alpha \\ 0 & 0 & -\alpha & \sqrt{1-\alpha^{2}}\end{array}\right)$

that can be interpreted (although this interpretation is unnecessary) as a rotation of an angle $\arcsin (\alpha)$ around a (two-dimensional) axis that leaves invariant the subspace corresponding to the $\{H\}$ non-degenerate eigenvalues.

Besides this, in this paper, the matrix $\{H\}$ in Table 3 has also been reordered with respect to Ref. [16] to respect also the symmetry between the $x$ and $y$ variables. This is a physical requirement because nothing in nature can distinguish between these two coordinates.

The resulting transformation matrix $\left\{T_{\alpha}\right\}$ is decomposed for presentation purposes into two summands $\left\{T_{\alpha}\right\}=\left\{T_{\alpha A}\right\}+\left\{T_{\alpha, B}\right\}$ which appear in Table 4 . It is to be stressed that $\left\{T_{\alpha A}\right\}$ and $\left\{T_{\alpha B}\right\}$ are not transformation matrices: they are not unitary. They are 
also used to separate the elements of $\left\{T_{\alpha}\right\}$ into terms that transform according to different irreducible representations in the group $\mathrm{D}_{4 \mathrm{~h}}$. Note that when $\alpha=0,\left\{T_{\alpha B}\right\}$ vanishes and $\left\{T_{\alpha A}\right\}$ becomes equal to the matrix $\{T\}$ appearing in Table 3 once normalized with the row norms of this matrix, $N_{1}, N_{2}, N_{3}$, and $N_{4}$. Some terms disappear in both matrices for $\alpha=1$.

The denominators $N_{1}, N_{2}, N_{3}$, and $N_{4}$ are invariant under all the $\mathrm{D}_{4 \mathrm{~h}}$ operations. They contain groups of variables that follow the identical representation $A_{1}$, as apparent in Table 2 or constants that are also invariant. Again by inspection of Table 2, the numerators of matrices $\left\{T_{\alpha A}\right\}$ and $\left\{T_{\alpha B}\right\}$ follow the irreducible representations presented in Table 5.

\subsection{The diagonalized Hamiltonian}

This topic has already been properly studied [16]. A simple Schrödinger equation can be written as

$-\frac{\hbar^{2}}{2 m^{*}} \nabla^{2} \Phi+U \Phi=\left(E-E^{*}\right) \Phi$

Table 5

Irreducible representations followed by the elements of the $\left\{T_{\alpha A}\right\}$ and $\left\{T_{\alpha B}\right\}$ matrices.

$\Gamma\left(T_{\alpha A}\right)=\left(\begin{array}{cccc}E_{u} & E_{u} & A_{2 u} & A_{1 g} \\ E_{u} & E_{u} & A_{2 u} & A_{1 g} \\ E_{u} & E_{u} & 0 & 0 \\ E_{g} & E_{g} & A_{1 g} & 0\end{array}\right) \quad \Gamma\left(T_{\alpha B}\right)=\left(\begin{array}{cccc}0 & 0 & A_{1 g} & A_{2 u} \\ 0 & 0 & A_{1 g} & A_{2 u} \\ E_{g} & E_{g} & A_{1 g} & A_{1 g} \\ E_{u} & E_{u} & 0 & A_{1 g}\end{array}\right)$

The asterisk in the mass refers to the effective mass to be used for each band: split-off (so), heavy holes $(h h)$, light holes $(l h)$ and conduction band $(c b) . E$ is the eigenvalue energy and $E^{*}$ is the origin of energies for each case, situated at the edge of the corresponding band. $U$ is the potential offset caused by the nanostructure. In the case of holes, its sign must be reversed and the effective mass taken as being positive. Notice that, in this paper, VB and CB energy levels refer to the extended states in the valence and conduction bands of the barrier material (GaAs) whereas $h h, l h$, so and $c b$ refer to the confined states created by the QDs and their energy can be located within the barrier material bandgap.

This equation is not different from the one used for the single band k.p method, used widely. For a squat box structure, a separation of variables method can be accurately used [12]. In this case, the confined solutions take the form of the product of three harmonic functions (even or odd) in $x, y$ and $z$ within the QD potential well flanked by fading exponentials outside it. These are labeled by three quantum numbers (QNs) each one corresponding to the one-dimensional solution chosen, from less to more energetic; for example, the eigenfunction can be labeled $c b(1,2,1)$. Odd QNs refer to even one-dimensional solutions and vice versa.

We reproduce in Fig. 1 the energy levels corresponding to the quantum dots described as sample SB in reference [22]. As a consequence of the large effective mass, there are many $h h$ eigenvalues. A density of states rather than the energy levels is presented in this case. The data in this figure corresponds to the cell labeled SB in [22] and details about its fabrication are given in that reference. It is a QD-IBSC device grown by MBE on a GaAs

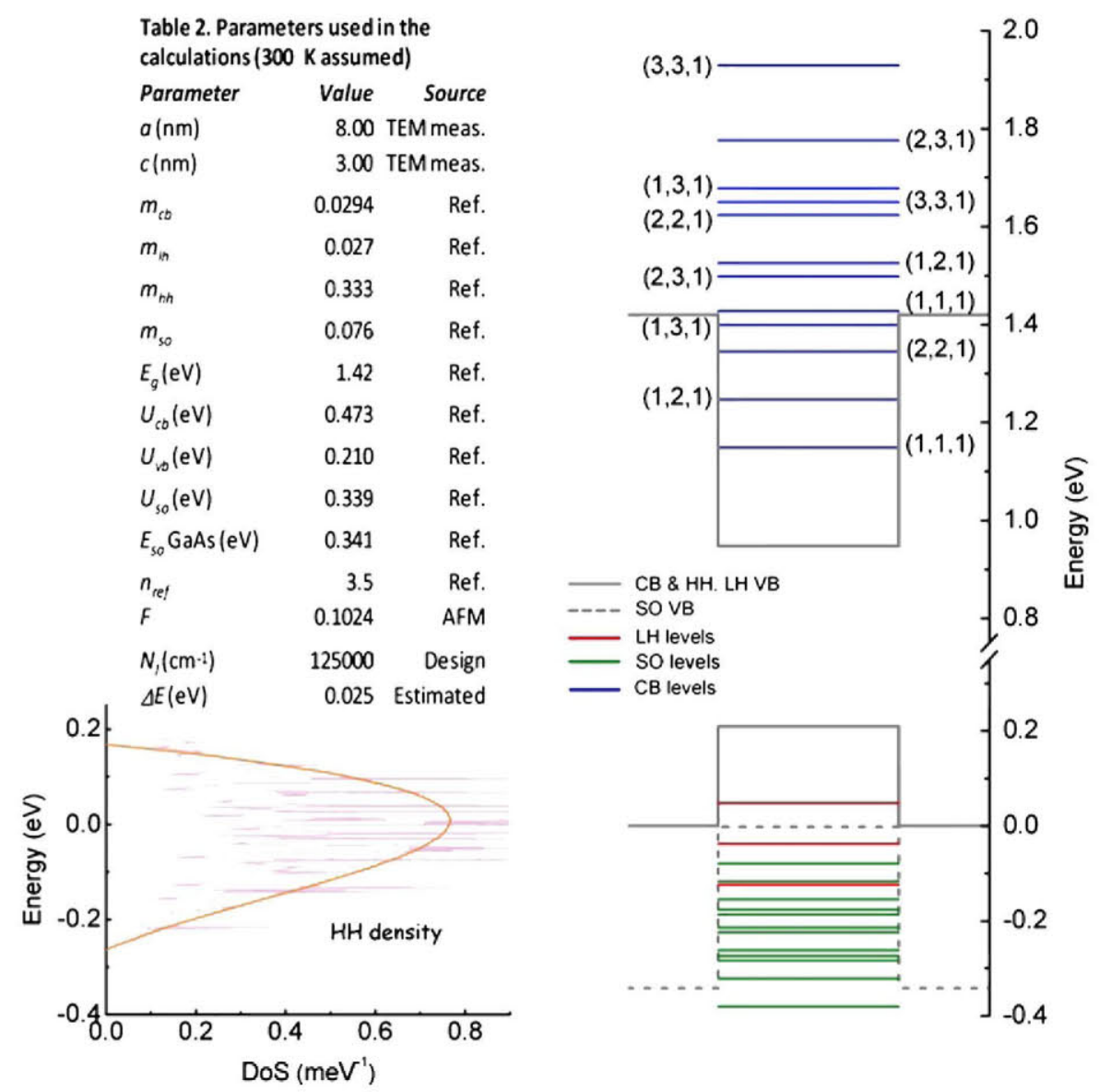

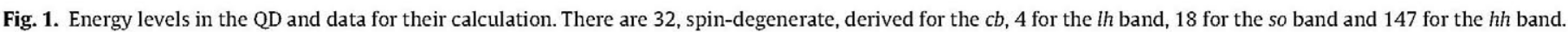

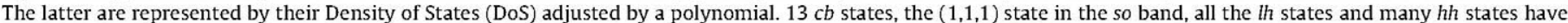

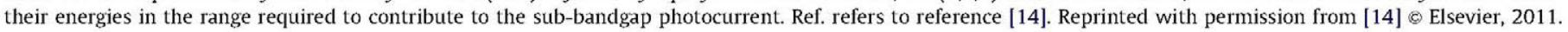



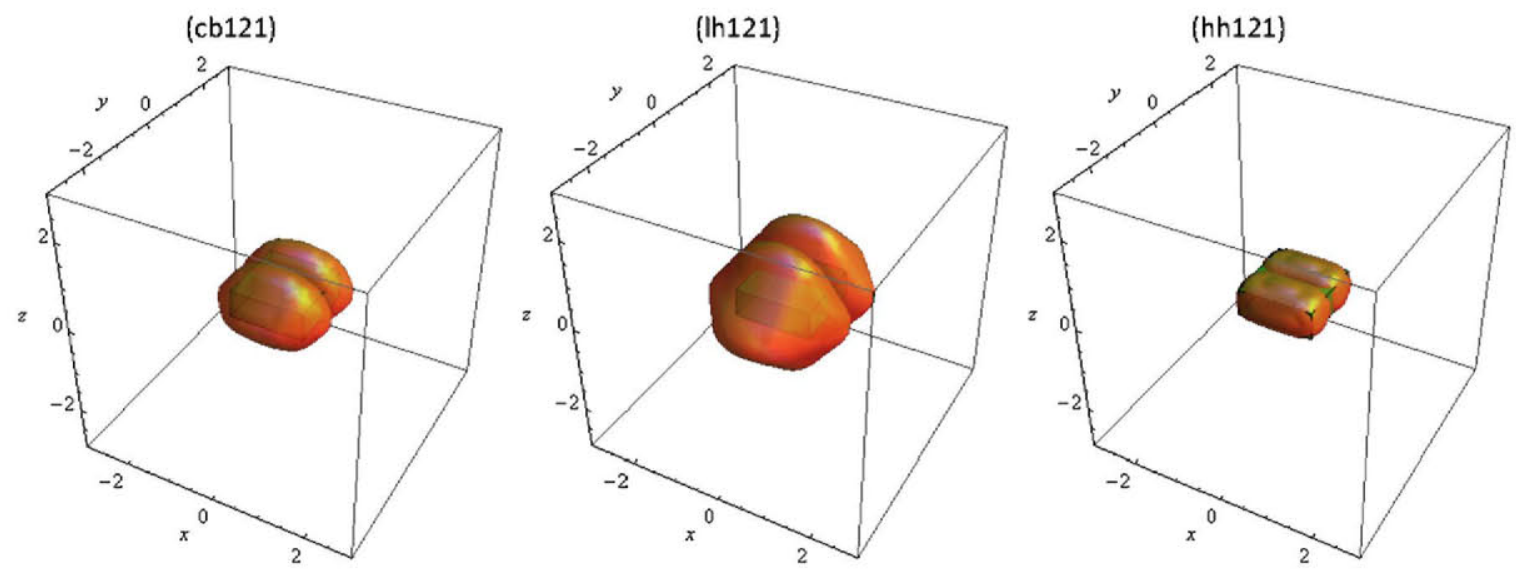

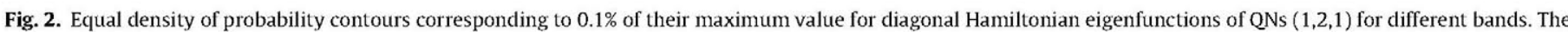
green box represents the QD. Space coordinates are in unities of $d(d=10 \mathrm{~nm})$.

wafer. First the structure contains a n-type buffer topped by a $\mathrm{GaAlAs}$ back surface field structure. Then the IB region is formed by a stacking of 30 self-assembled InAs QD layers built up by Stranski-Krastanov (SK) growth mode and separated by thick $(80 \mathrm{~nm})$ GaAs spacers, which are meant to dilute the accumulated vertical strain and thus prevent dislocations. This QD stacking strategy is an alternative to the strain compensation technique $[6,7,10,23]$ and also prevents carrier tunneling between contiguous QD layers [22]. Si $\delta$-doping was added within each of the QD layers to partially fill the IB with electrons [2]. The structure is capped with an ordinary p-type GaAs emitter and a passivating InAlGa layer for surface. An anti-reflection coating is on top.

Fig. 2 represents the spread of the wavefunctions for the $(1,2,1)$ QNs corresponding to the $c b$ the $l h$ and the $h h$ band. Notice that the high effective mass of the hhs makes the electron stay strongly confined within the QD. On the contrary, the lh escapes largely from it because of the light effective mass and the weak confining potential. The $c b$ electron is more confined due to the stronger potential.

\subsection{Symmetry of the diagonalized Hamiltonian eigenfunctions}

Let us consider first the function $(1,1,1)$. It is the product of three even one-dimensional functions, one for each coordinate. Furthermore, the functions for $x$ and for $y$ are exactly the same. Therefore, applying the symmetries, the three-dimensional eigenfunction is always invariant (see in Table 1 how the variables are transformed by the different symmetry operations). They follow the identical representation $A_{1 g}$. The same will happen for any eigenfunction whose QNs are of the type (odd1,odd1,odd), the first two corresponding to the same one-dimensional function, in this case, of even symmetry. The z-eigenfunction is always different because in this direction the potential well has a width $2 c \neq 2 a=2 b$.

With the same arguments, looking again to Table 1, we can see how the eigenfunctions with QNs in the second, third and fourth lines of Table 6 are transformed. The symmetry operations leave the eigenfunctions unchanged (character +1 ) or changes their sign (character -1 ) and they all follow different one-dimensional $\left(\chi_{E}=1\right)$ irreducible representations of the symmetry group.

For the case of $(1,2,1)$, the level is doubly degenerate and the (Hilbert space) basis functions are $(1,2,1)$ and $(2,1,1)$. The representation is two-dimensional $\left(\chi_{E}=2\right)$ and the eigenfunction $(1,2,1)$ may correspond to the representation column vector $\{1,0\}$ and the eigenfunction $(2,1,1)$ to the column vector $\{0,1\}$. For, e.g., the symmetry operation $I C_{4}$, which transforms the point
Table 6

Representation associated to the diagonalized Hamiltonian eigenfunctions.

One-dimensional quantum numbers (parity Colum vectors for 1D of the function opposite to parity of $\mathrm{QN}$ ) representations

(odd1, odd 1 , odd)

(odd1, odd1, even)

(even 1 , even 1 , odd)

(even 1 , even 1 , even)

(odd1, even2, odd), (even1, odd2, odd)

(odd1, even2, even), (even1, odd2, even)

(odd1, odd2, odd)

(odd1, odd2, even)

(even1, even2, odd)

(even1, even2, even)

$\begin{array}{ll} & A_{1 g} \\ & A_{2 u} \\ & B_{1 g} \\ & B_{2 u} \\ & E_{u} \\ & E_{g} \\ & A_{1 g}+B_{2 g} \\ \{1,1\},\{1,-1\} & A_{2 u}+B_{1 u} \\ \{1,1\},\{1,-1\} & A_{2 g}+B_{1 g} \\ \{1,-1\},\{1,1\} & A_{1 u}+B_{2 u}\end{array}$

$(x, y, z)$ into $(-y, x,-z)$, the eigenfunction $(1,2,1)$ is transformed into $(2,1,1)$ and $(2,1,1)$ into $-(1,2,1)$, in other words to the representation vector $\{1,0\}$ corresponds $\{0,1\}$ and to the vector $\{0,1\}$ corresponds the vector $\{-1,0\}$. The matrix of this transformation appears in Table 7 under the $I C_{4}$ symmetry operation. We can repeat this procedure for the rest of the symmetry operations. The transformation matrices are also in Table 7 . The characters (which are the traces of the matrices) correspond to the twodimensional irreducible representation $E_{u}$, as presented in Table 6 . The preceding arguments are also applicable to the eigenfunction of the following line in Table 6 whose representation is $E_{g}$.

The procedure above can also be applied to the (odd1,odd2,odd) eigenfunctions. They also generate two-dimensional representations and the corresponding matrices may be deduced along with the table of characters of the representation. However, they are not irreducible (the sum of the square of the characters is not $N_{G}=16$ but 32). By application of Eq. (2), they are found to be the direct sum of two one-dimensional irreducible representations. This appears in the corresponding Table 6 , line 7 . In the column labeled "Column vector for 1D representations", the vectors leading to these onedimensional representations are registered. In the case we are examining, the $\{1,1\}$ and $\{1,-1\}$ vectors correspond to the eigenfunction (odd 1 , odd 2 ,odd) + (odd2, odd 1 , odd) and (odd1, odd2, odd) (odd2,odd1,odd). It can be seen that these eigenfunctions transform into themselves, with or without a change of sign, so reflecting the fact that they span a one-dimensional representation. The same happens for all the symmetry combinations in the Table 6 , lines 8 , 9 and 10 . 
Table 7

Matrices of the two-dimensional representation subtended by the eigenfunctions (odd1,even2,odd) and (even2,odd1,odd).

\begin{tabular}{|c|c|c|c|c|c|c|c|}
\hline$E$ & $I C^{\prime \prime} \times 2$ & $I C^{\prime}{ }_{y 2}$ & $I C_{2}$ & $I$ & $C_{x 2}^{\prime}$ & $C_{y 2}^{\prime \prime}$ & $C_{2}$ \\
\hline$+x_{1}+y_{1}+z$ & $-x_{1}+y_{1}+z$ & $+x,-y_{1}+z$ & $+x,+y,-z$ & $-x,-y,-z$ & $+x,-y,-z$ & $-x,+y,-z$ & $-x,-y,+z$ \\
\hline$\left(\begin{array}{ll}1 & 0 \\
0 & 1\end{array}\right)$ & $\left(\begin{array}{cc}1 & 0 \\
0 & -1\end{array}\right)$ & $\left(\begin{array}{cc}-1 & 0 \\
0 & 1\end{array}\right)$ & $\left(\begin{array}{ll}1 & 0 \\
0 & 1\end{array}\right)$ & $\left(\begin{array}{cc}-1 & 0 \\
0 & -1\end{array}\right)$ & $\left(\begin{array}{cc}-1 & 0 \\
0 & 1\end{array}\right)$ & $\left(\begin{array}{cc}1 & 0 \\
0 & -1\end{array}\right)$ & $\left(\begin{array}{cc}-1 & 0 \\
0 & -1\end{array}\right)$ \\
\hline$I C_{d s 2}^{\prime}$ & $-C_{4}$ & $C_{4}$ & $C_{d p 2}^{\prime}$ & $C_{d s 2}$ & $I\left(-C_{4}\right)$ & $I C_{4}$ & $I C_{d p 2}^{\prime}$ \\
\hline$+y,+x,+z$ & $-y,+x,+z$ & $+y,-x,+z$ & $+y,+x,-z$ & $-y,-x,-z$ & $+y,-x,-z$ & $-y,+x,-z$ & $-y,-x,+z$ \\
\hline$\left(\begin{array}{ll}0 & 1 \\
0\end{array}\right)$ & $\left(\begin{array}{ll}0 & -1 \\
)\end{array}\right)$ & $\left(\begin{array}{ll}0 & 1\end{array}\right)$ & $\left(\begin{array}{ll}0 & 1 \\
1\end{array}\right)$ & $\left(\begin{array}{ll}0 & -1\end{array}\right)$ & $\left(\begin{array}{ll}0 & 1\end{array}\right)$ & $\left(\begin{array}{ll}0 & -1\end{array}\right)$ & $\left(\begin{array}{ll}0 & -1\end{array}\right)$ \\
\hline$\left(\begin{array}{ll}1 & 0\end{array}\right)$ & $\left(\begin{array}{ll}1 & 0\end{array}\right)$ & $\left(\begin{array}{ll}-1 & 0\end{array}\right)$ & $\left(\begin{array}{ll}1 & 0\end{array}\right)$ & $\left(\begin{array}{ll}-1 & 0\end{array}\right)$ & $\left(\begin{array}{ll}-1 & 0\end{array}\right)$ & $\left(\begin{array}{ll}1 & 0\end{array}\right)$ & $\left(\begin{array}{ll}-1 & 0\end{array}\right)$ \\
\hline
\end{tabular}

\subsection{The envelope functions}

With the nomenclature in this paper, i.e., $\Phi_{u, \mathbf{n}}=\Delta \Omega^{1 / 2} \Phi_{u}(\mathbf{n} L / N)$ and $\varphi_{u, \mathbf{k}}=\varphi_{u}(2 \pi \mathbf{\kappa} / L)$ with $u$ referring to the $c b, l h, h h$, or so band, the diagonalized eigenfunctions can also be expressed by their DFT, that is,

$\varphi_{u, \mathbf{\kappa}}=\frac{1}{N^{3 / 2}} \sum_{\mathbf{n}} \Phi_{u, \mathbf{n}} e^{-2 \pi i \mathbf{k} \cdot \mathbf{n} / N}$

so that $\left\{\psi_{\mathbf{\kappa}}\right\}=\left\{T_{\alpha}^{+}\left(2 \pi \kappa_{x} d / L, 2 \pi \kappa_{y} d / L, 2 \pi \kappa_{z} d / L\right)\right\}\left\{\varphi_{\boldsymbol{\kappa}}\right\}=\left\{T_{\alpha, \mathbf{k}}^{+}\right\}\left\{\varphi_{\mathbf{k}}\right\}$ [16]. In this expression, $\left\{\psi_{\mathbf{k}}\right\}$ is a column vector of components $\psi_{v, \boldsymbol{\kappa}}$ (with $v$ referring to the associated involute) and $\left\{T_{\alpha, \boldsymbol{k}}^{+}\right\}$, of terms $T_{\alpha, \mathbf{k}}^{+(v, u)}$, is the Hermitical conjugate of the square matrix described in Table 4, associated to the projection onto the involutes of states of different bands. In fact, for a given eigenvalue, only one band is involved and a single term of the column vector $\left\{\varphi_{\mathbf{K}}\right\}$ is not zero so that $\psi_{v, \boldsymbol{\kappa}}=T_{\alpha, \boldsymbol{\kappa}}^{(u, v)} \varphi_{u, \boldsymbol{\kappa}}$ (note that the Hermitic conjugation is performed by permuting the indices). Once $\psi_{v, \boldsymbol{\kappa}}$ is known, $\Psi_{v \cdot \mathbf{n},}$ can be obtained by application of the IDFT. Note that the terms $T_{\alpha, \mathbf{k}}^{(u, v)}$ are to be calculated at the coordinates $\left(2 \pi \kappa_{x} d / L, 2 \pi \kappa_{y} d / L, 2 \pi \kappa_{z} d / L\right)$. The ratio $d / L$ is to be used because $T_{\alpha}^{+}$appears as a function $T_{\alpha}^{+}\left(K_{x}, K_{y}, K_{z}\right)$ in Table 4 and not of $\left(k_{x}, k_{y}, k_{z}\right)$.

In summary, in the EKPH, the calculation of the envelope function proceeds along the following scheme [16]: (a) the diagonalized Hamiltonian eigenfunction is calculated; (b) its DFT is calculated; (c) it is multiplied (term by term for each $\mathbf{K}$ ) by the corresponding matrix $\left\{T_{\alpha, k}^{+}\right\}$element; (d) the IDFT is calculated. The rows of the $\left\{T_{\alpha, \boldsymbol{\kappa}}\right\}$ matrix (the columns of $\left\{T_{\alpha, \boldsymbol{k}}^{+}\right\}$) correspond to the different eigenvalues $(1,2,3$ and 4 for the $c b, l h$, $h h$ and $s o$, respectively). The columns refer to the projections on the $X-, Y$-, $Z$ - and $S$-involutes.

An important property of the k.p method [15] (at least for large nanostructures) is that integrals of slow varying functions multiplied by fast varying functions are split into the product of the integrals of the slow and fast varying functions calculated separately. Applying this property,

$$
\begin{aligned}
& \langle\Xi(\mathbf{r}) \mid \Xi(\mathbf{r})\rangle=1 \\
& \quad=\sum_{\nu, v^{\prime}}\left\langle u_{0, v}(\mathbf{r}) \mid u_{0, v^{\prime}}(\mathbf{r})\right\rangle\left\langle\Psi_{v}(\mathbf{r}) \mid \Psi_{v^{\prime}}(\mathbf{r})\right\rangle=\sum_{v}\left\langle\Psi_{v}(\mathbf{r}) \mid \Psi_{v}(\mathbf{r})\right\rangle
\end{aligned}
$$

The later equality makes use of the orthonormal properties of the set $\left|u_{0, v}(\mathbf{r})\right\rangle$. The integral $\left\langle\Psi_{v}(\mathbf{r}) \mid \Psi_{v}(\mathbf{r})\right\rangle$ can be considered the projection of $|\Xi(\mathbf{r})\rangle$ on the corresponding involute. In the discretized scheme used, Eq. (11) is written as the sum (note that, by definition, $\Psi_{v, \mathbf{n}}^{*} \Psi_{v, \mathbf{n}}$ contains an element of volume, so that the sums are approximate integrals)

$$
\begin{array}{r}
\sum_{v}\left\langle\Psi_{v}(\mathbf{r}) \mid \Psi_{v}(\mathbf{r})\right\rangle=\sum_{v, \mathbf{n}} \Psi_{v, \mathbf{n}}^{*} \Psi_{v, \mathbf{n}}=\sum_{v, \mathbf{K}} \psi_{v, \mathbf{\kappa}}^{*} \psi_{v, \mathbf{K}} \\
=\sum_{v, \mathbf{k}} T_{\alpha, \mathbf{K}}^{(u, v)} T_{\alpha, \mathbf{K}}^{*(u, v)} \varphi_{u, \mathbf{\kappa}}^{*} \varphi_{u, \mathbf{\kappa}}=\sum_{\mathbf{K}} \varphi_{u, \mathbf{\kappa}}^{*} \varphi_{u, \mathbf{K}}=1
\end{array}
$$

The second equality describes a fundamental property of the DFT. The fourth equality derives from the fact that the rows of $\left\{T_{\alpha, \boldsymbol{\kappa}}\right\}$ are normalized for any value of $\boldsymbol{\kappa}$ (with one exception to be discussed). In the last equality we express that $\Phi_{\mathbf{n}}$ has been normalized.

Fig. 3 shows the values of the $\left\{T_{\alpha, \boldsymbol{k}}\right\}$ terms over a range of $K$ values. The normalization of the $\left\{T_{\alpha, \boldsymbol{\kappa}}\right\}$ rows is not always fulfilled for all the $\boldsymbol{\kappa}$. In fact, $\left\{T_{\alpha}\left(0,0, K_{z}\right)\right\}$ is not defined for the $l h$, $h h$ or so bands, as can be observed in Fig. 3. It takes different value depending from which direction the origin of the $z$-constant plane is approached. This indetermination is also visible in Table 4 . In this paper the value of $\left\{T_{\alpha}\left(0,0, K_{z}\right)\right\}$ is defined as the average of the limit when $h \rightarrow 0$ of $\left\{T_{\alpha}\left( \pm h, \pm h, K_{z}\right)\right\}$. Furthermore, for the case $\left\{T_{\alpha}(0,0,0)\right\}$, we also take the average of $\left\{T_{\alpha}( \pm h, \pm h, \pm h)\right\}$. This definition is symmetric with respect the $D_{4 h}$ operations but it is arbitrary. Because of this, The normalization of the $\left\{T_{\alpha, \mathbf{\kappa}}\right\}$ rows is not always fulfilled for $\boldsymbol{\kappa}=\left(0,0, \mathcal{K}_{z}\right)$. Such singularity is only transferred to the envelopes when the $x$ - and $y$ - functions are both even (QNs odd) and become negligible when the QNs are of high order. Furthermore, it also becomes negligible when the calculation cube size is made big enough (for $h h(1,1,1)$ the sum of the terms of Eq. (11) is 0.9452 for a cube side of $6 d$ and 0.9964 for $24 d)$. Nevertheless, even if the difference from one is not totally negligible, a renormalization is performed by multiplying all the envelope functions by a factor such that Eq. (11) is fulfilled (the sum of projections becomes unity).

The envelope functions corresponding to the diagonalized Hamiltonian solutions in Fig. 2 are presented in Fig. 4 for a rotation of $\left\{T_{\alpha, \boldsymbol{\kappa}}\right\}$ with $\alpha=0.3$.

The $c b Z$-envelope appears in the corners of the calculation cube. In all cases, it has a negligible overlap with the $t h$ or $h h$ envelopes. Furthermore, if the cube is enlarged (even from a $6 d$ to a $24 d$ side) the envelope function changes its position and is situated again at the cube corners. We deduce this is an artifact (associated to the periodic conditions at the cube faces: the electron in the corner "sees" eight potential wells at the same distance of which only one is real) and therefore we discard it. In consequence, a new renormalization is made by multiplying the $X$-, $Y$ - and $S$-envelope functions in the $c b$ by a factor that allows Eq. (11) to be fulfilled once the $Z$-envelope is discarded. This factor is around 1.1 for $\alpha=0.3$ and seems fairly independent of the cube size.

We represent in Fig. 5 the projection onto the different involutes of the $c b(1,1,1)$ and the $h h(1,2,1)$ states (the states involved in the sub-bandgap absorption threshold). Solid lines refer to the transformation matrix studied so far: the row corresponding to the $h h$ states is the third in Table 4 . We call it the standard configuration. We observe that the $c b$ state is almost totally projected onto the $S$ involute (around 96\%); the remaining envelopes are small but not negligible. It is mainly a $c b$ state and this justifies the commonly used one-band approximation to study it. This statement is thought to be valid for other $c b$ states. For low $\alpha$, the $h h$ state is essentially projected onto the $X$ and $Y$ involutes and to a lesser extent onto the $Z$ involute. The projection onto the $S$ involute is very small for $\alpha<0.3$. This means that the $h h$ state is almost totally projected onto the 
(cb)

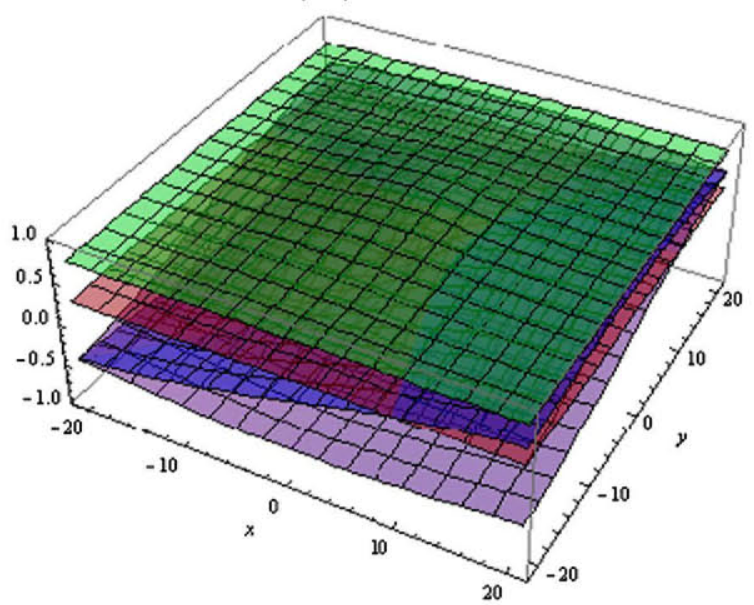

(hh)

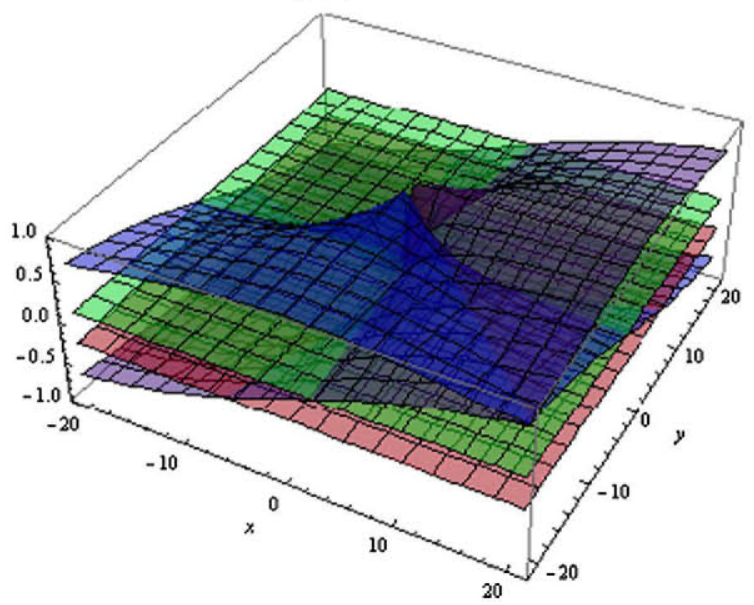

(Ih)

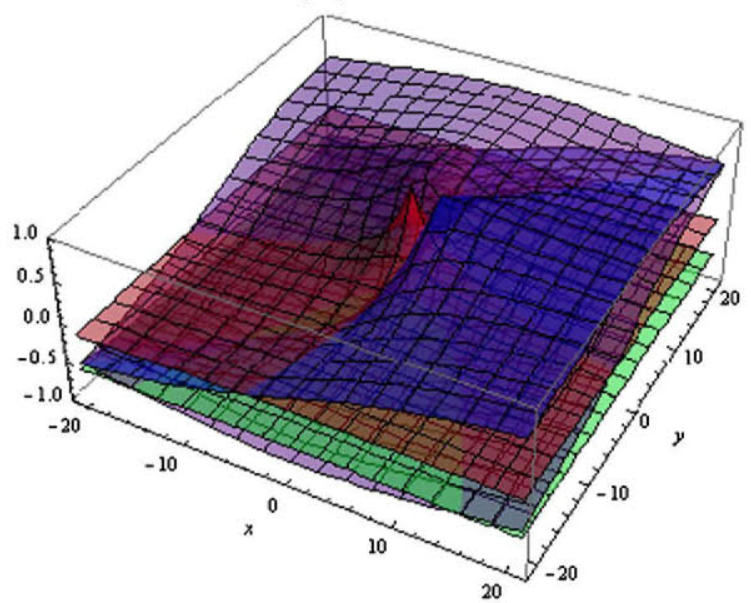

(so)

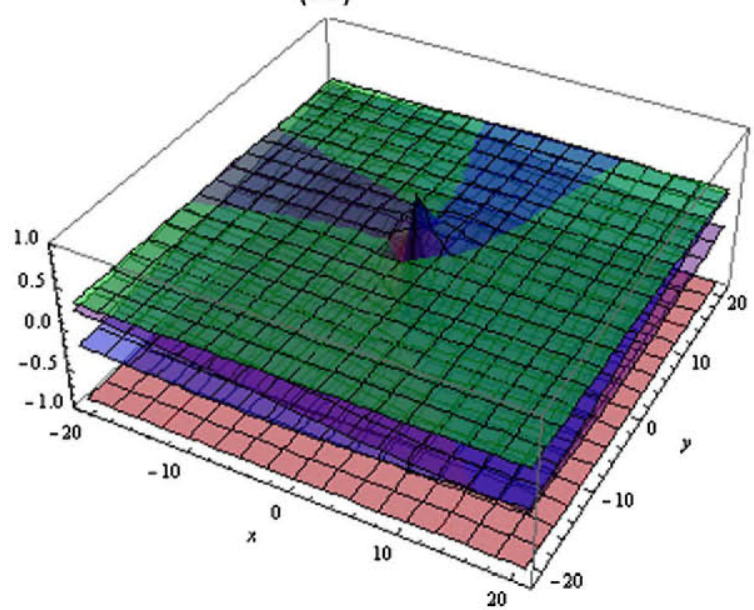

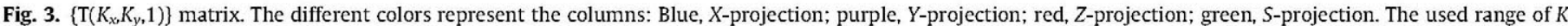

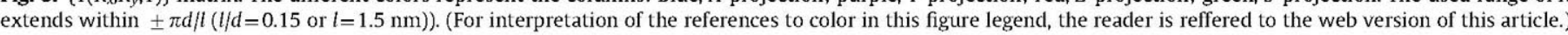

$X, Y, Z$ involutes (that are eigenvalues of the $\mathrm{VB}$ ). For $\alpha>0.3$ the projection onto the $S$-involute is significant and we may say (somewhat improperly) that the wavefunction contains VB and $\mathrm{CB}$ characteristics. If the state $h h(1,2,1)$ is changed into $h h(2,1,2)$, the projection onto the $X$ - and $Y$-envelopes are interchanged, as expected. The rest remains unchanged.

The standard configuration, which associates the third row in Table 4 to the $h h$ eigenvalue (remember that rows and columns are interchanged in the transposed matrix) and the forth to the so eigenvalue, is arbitrary. The opposite option might have been chosen and the fourth row might have been associated to the $h h$ eigenfunction. This is called the interchanged configuration. This is presented in Fig. 5 using dashed lines. Again for low $\alpha$ s the projection on the $S$-involute is negligible.

\section{Symmetry considerations in the calculations of the optical elements of matrix}

\subsection{The photon-absorption elements of matrix}

The dipole matrix ruling the photon-electron interaction is.

$$
\begin{aligned}
& \langle\Xi|\boldsymbol{\varepsilon} \cdot \mathbf{r}| \Xi\rangle=\sum_{v, v^{\prime}}\left\langle u_{0, v} \mid u_{0, v^{\prime}}\right\rangle\left\langle\Psi_{v}|\boldsymbol{\varepsilon} \cdot \mathbf{r}| \Psi_{v^{\prime}}\right\rangle=\sum_{v}\left\langle\Psi_{v}|\boldsymbol{\varepsilon} \cdot \mathbf{r}| \Psi_{v}\right\rangle \\
& \quad=\left\langle\Psi_{S}|\boldsymbol{\varepsilon} \cdot \mathbf{r}| \Psi_{S}\right\rangle+\left\langle\Psi_{X}|\boldsymbol{\varepsilon} \cdot \mathbf{r}| \Psi_{X}\right\rangle+\left\langle\Psi_{Y}|\boldsymbol{\varepsilon} \cdot \mathbf{r}| \Psi_{Y}\right\rangle+\left\langle\Psi_{Z}|\boldsymbol{\varepsilon} \cdot \mathbf{r}| \Psi_{Z}\right\rangle
\end{aligned}
$$

where $\varepsilon$ is the light polarization vector. In this equation, the same approximation has been made as in Eq. (11) concerning the integration of slow and fast varying functions. Thus, under this approximation (not valid for very small QDs [24]) only the envelope functions play a role. Exact expression of the absorption coefficients in function of the matrix elements may be found in $[13 ; 14 ; 16]$. We can define the polarization by the Euler angles $(\phi, \theta)$ characterizing its direction. Thus

$\langle\Xi|\boldsymbol{\varepsilon} \cdot \mathbf{r}| \Xi\rangle=\cos \phi \sin \theta\langle\Xi|x| \Xi\rangle+\sin \phi \sin \theta\langle\Xi|y| \Xi\rangle+\cos \theta\langle\Xi|z| \Xi\rangle$

and the same for its components. We use the following nomenclature,

$$
\begin{aligned}
M x & =\langle\Xi|x| \Xi\rangle=\left\langle\Psi_{X}|x| \Psi_{X}\right\rangle+\left\langle\Psi_{Y}|x| \Psi_{Y}\right\rangle+\left\langle\Psi_{Z}|x| \Psi_{Z}\right\rangle+\left\langle\Psi_{S}|x| \Psi_{S}\right\rangle \\
& =M x X+M x Y+M x Z+M x S
\end{aligned}
$$

and similar for the $y$ and $z$ polarizations.

The absorption coefficient is proportional to the squared module of the matrix element. Furthermore, in many cases the light is not polarized and the average of a set of polarizations is to be taken. An interesting case is, for instance, vertical unpolarized light, in which case the absorption is proportional to $\left(|M x|^{2}+|M y|^{2}\right) / 2[14]$.

For symmetry reasons, many terms of the matrix elements are zero and group theory can be applied to spare their calculation, as is described in the following section. 


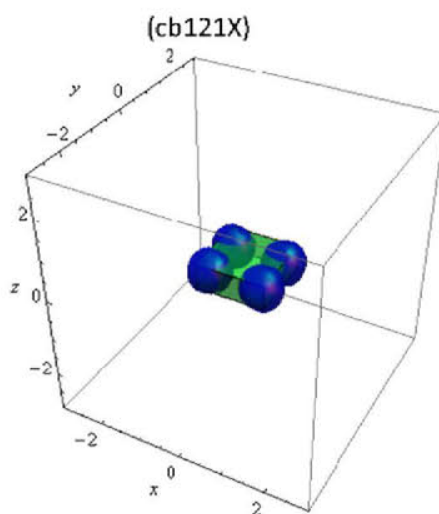

(cb121Y)
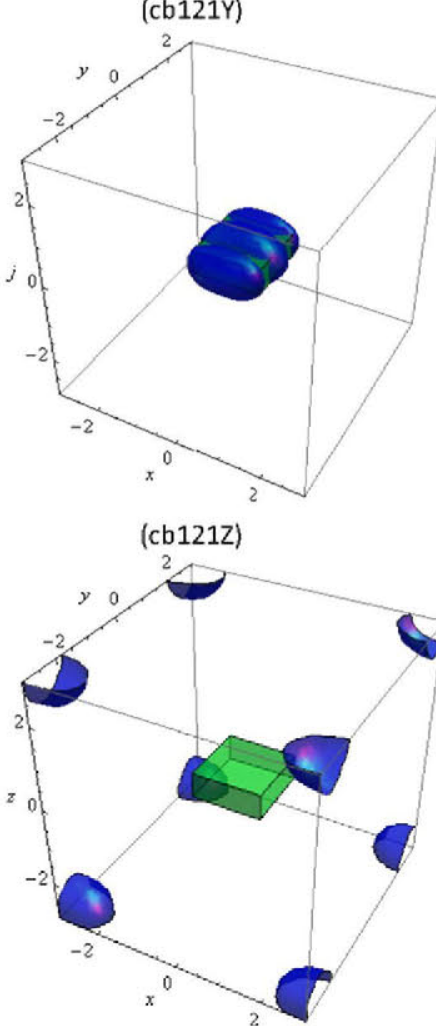

(cb1215)

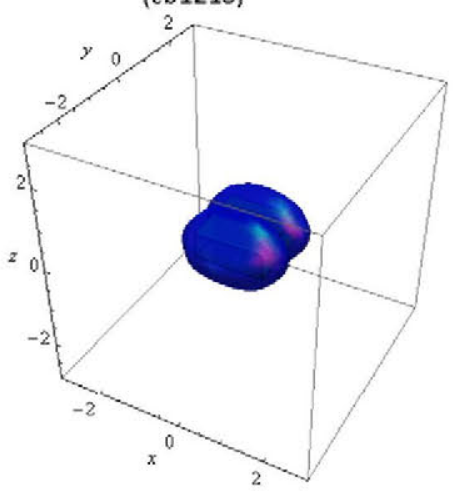

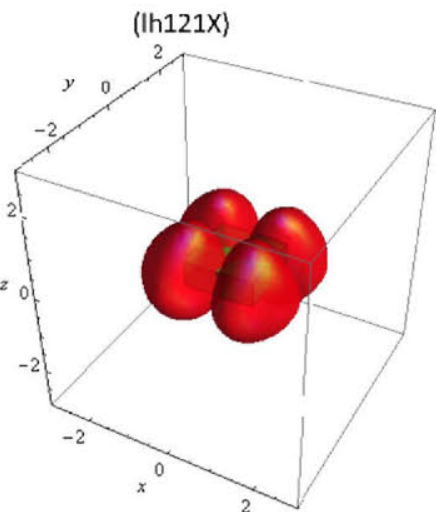

(Ih121Y)
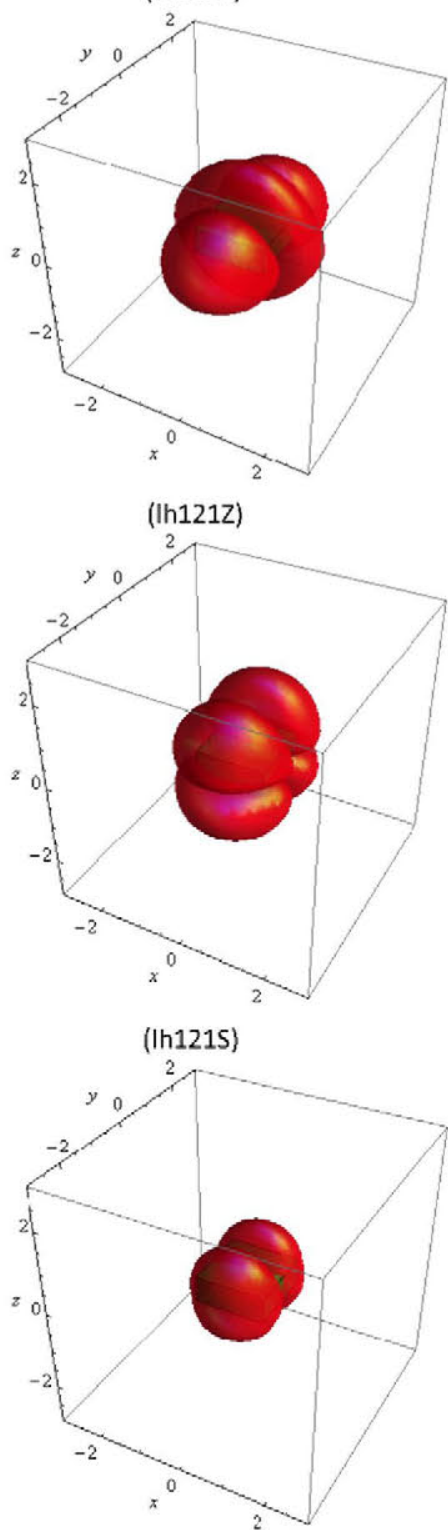

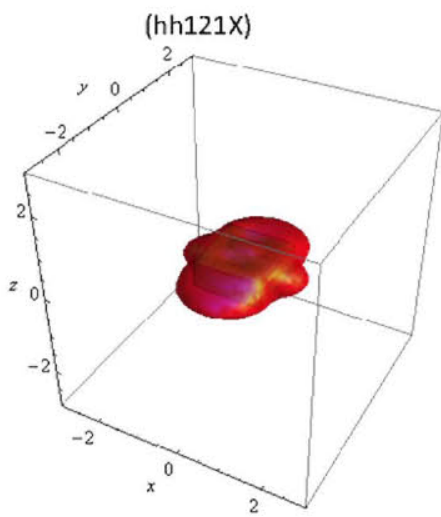

(hh121Y)
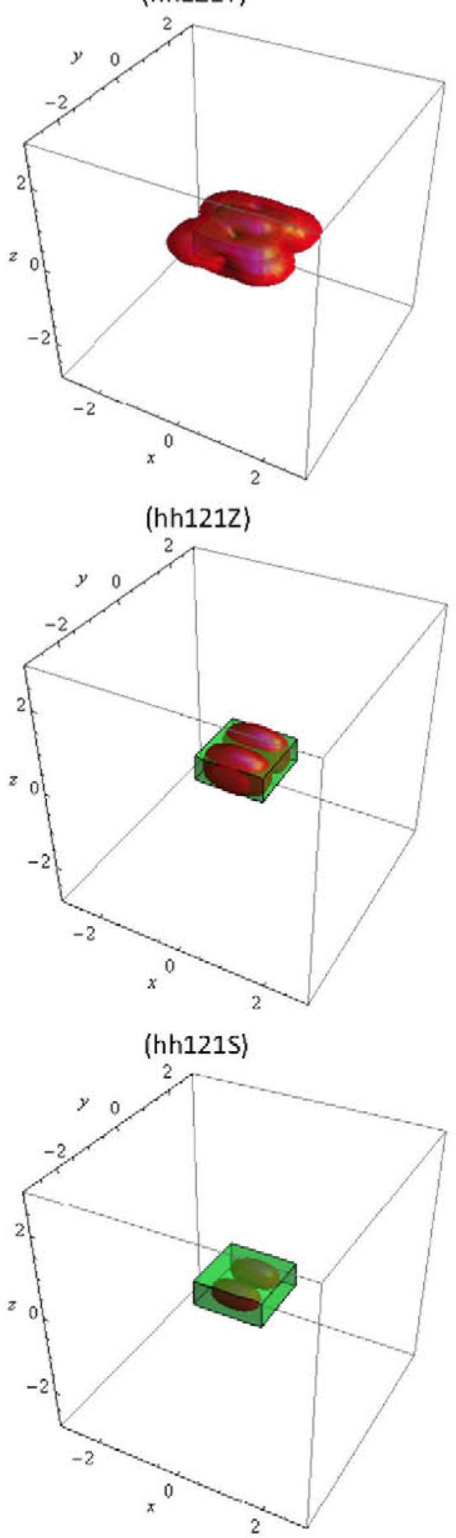

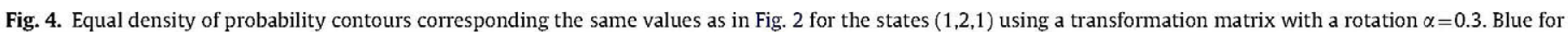

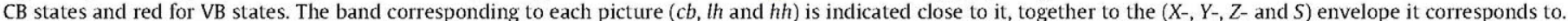

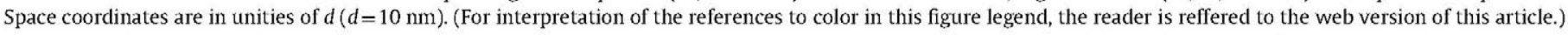

\subsection{Zero terms in the matrix elements}

For each symmetry operation, the characteristic of the Cartesian product of several representation matrices is the ordinary product of their characteristics. Additionally, the characteristic of the direct sum of several representation matrices is the ordinary sum of their characteristics.

The necessary condition for an operator element of matrix to be non-zero is that the Cartesian product representation of the 
operator and one of the two wavefunctions and the representation of the remaining wavefunction contain at least one irreducible representation in common. This is equivalent to saying that the Cartesian product of the two wavefunction representations and that of the operator both contain the identical representation at least once [19]; the matrix element will be zero if this is not the case. This can be easily verified by calculating the characters of the triple Cartesian product representation and by using Eq. (2) to calculate the number of times the identical representation is included. If the signed sum of these characters is zero (it is included zero times), the transition is forbidden. Otherwise, the result must be a multiple of $N_{G}$ (this can be used as a check) and the transition is not forbidden by symmetry considerations, but the matrix element can still be zero.

Let us apply this procedure to the calculation of any of the terms of the type of those in Eq. (15). The operator will be $x, y$ or $z$ depending on the polarization. Their associated representations are (see Table 2) $E_{u}, E_{u}$ or $A_{2 u}$, respectively. Each envelope function is calculated by (a) obtaining a diagonalized eigenfunction, whose associated representations are in Table 6 (in some cases the representation is the direct sum of two irreducible

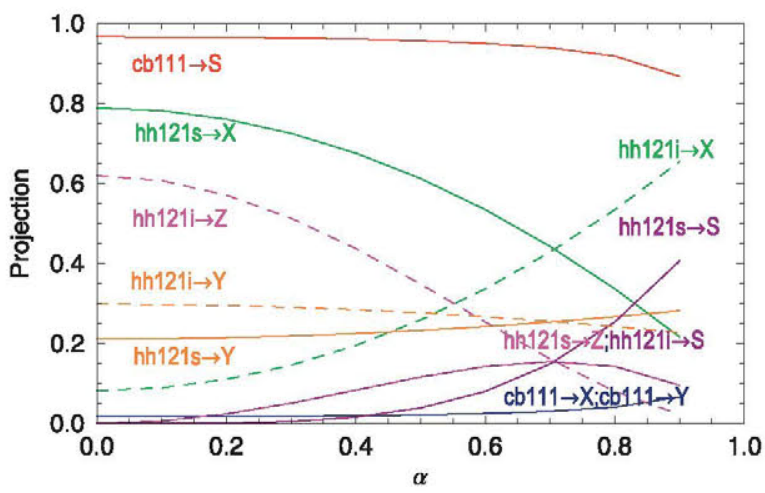

Fig. 5. Projection on the involutes of the $c b(1,1,1)$ and $h h(1,2,1)$ states. Solid lines, standard configuration (a " $s$ " appears in the labels). Dashed lines, interchanged configuration (an "i" appears in the labels). representations), (b) obtaining its DFT, associated to the same representation (Section 2.3), (c) multiplying it by a $T_{\alpha, \mathbf{k}}^{(u, v)}$ term, whose associated representation is again the direct sum of the representations of the terms $T_{\alpha A, \kappa}^{(u, v)}$ and $T_{\alpha B, \mathbf{k}}^{(u, v)}$, both appearing in Table 5 and finally (d) calculating the IDFT that also follows the same representation. This operation has to be done for the "initial" and "final" wavefunctions intervening in the dipole matrix element.

In summary the sum (on symmetry operations, not on classes)

$\sum_{G} \chi_{G}^{\Phi \text { ini }} \chi_{G}^{\text {Tini }} \chi_{G}^{M} \chi_{G}^{\Phi \text { fin }} \chi_{G}^{\text {Tfin }} / N_{G}=h^{\left(A_{i g}\right)}$

is to be performed and checked if it is zero. The superscripts ini and fin refer, respectively, to the initial and final eigenfunctions intervening and $M$ to the element of matrix, $x, y$ or $z$. For characters bearing the superindex $M$ and for many QN combinations associated with superindices $\Phi$ ini and $\Phi$ fin, the characters correspond to irreducible representations; in most $T$-matrix elements and in some QN combinations they are the direct sum of two irreducible representations. The calculation is very easy and can be performed by hand or programmed (even in a spreadsheet).

To have an idea of the calculation burden reduction, of the 1728 different types of matrix elements when classified by symmetry, 976 of the $h h \rightarrow c b$ transitions are forbidden by symmetry reasons and 1180 of the $l h \rightarrow c b$. For $\alpha=0$ the forbidden transitions are 1524 for the $h h \rightarrow c b$ transitions and 1352 for the $l h \rightarrow c b$.

Some of the transitions permitted by symmetry still have zero probability. This is the case, in particular, for all the transitions involving $Z$-envelope, supporting our statement that the $Z$ component of the cb states is an artifact and sustaining the normalization described above (note that this normalization does not affect the zeroes when calculating matrix elements).

By examining all the symmetry cases, we can extract the rules in Table 8. Note that these rules are the same for the $h h \rightarrow c b$ and the $s o \rightarrow c b$. This means they are not affected by the row (3 or 4 ) of Table 4 used for calculating the $h h$ and the so envelopes.

Note that, for the case of $\varepsilon=0$ and $\varepsilon=1$, many or all of the reducible representations in the matrix $T_{\alpha, \mathbf{k}}^{(u, v)}$ become irreducible

Table 8

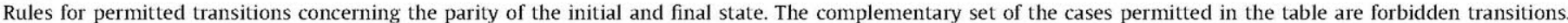
Permitted transitions may still have a zero matrix element.

\begin{tabular}{|c|c|c|c|}
\hline Transition & Polarization & Envelopes & Parity of initial and final states \\
\hline \multirow{3}{*}{$\begin{array}{l}h h \rightarrow c b \\
s o \rightarrow c b\end{array}$} & Horizontal $(x, y)$ & All & $\begin{array}{l}\text { - Different for one and only one of the } x \text { - and } y \text {-QNs } \\
\text { - Irrelevant for the } z \text {-QN }\end{array}$ \\
\hline & \multirow[b]{2}{*}{ Vertical $(z)$} & $X, Y$ & $\begin{array}{l}\text { - Either the same or different simultaneously for both } x \text { - and } y \text {-QNs } \\
\text { - Irrelevant for the } z-Q N\end{array}$ \\
\hline & & $Z, S$ & $\begin{array}{l}\text { - The same for both } x \text {-and } y \text {-QNs or the initial parity of the } x \text {-QN is the same } \\
\text { as the final parity of the } y \text {-QN and vice versa } \\
\text { - Irrelevant for the } z-\mathrm{QN}\end{array}$ \\
\hline \multirow{4}{*}{$l h \rightarrow c b$} & \multirow[b]{2}{*}{ Horizontal $(z, y)$} & $X, Y$ & $\begin{array}{l}\text { - Different for one and only one of the } x \text { - and } y \text {-QNs } \\
\text { - The same for the } z-\mathrm{QN}\end{array}$ \\
\hline & & $Z, S$ & $\begin{array}{l}\text { - Different for one and only one of the } x \text { - and } y \text {-QNs } \\
\text { - Irrelevant for the } z-\mathrm{QN}\end{array}$ \\
\hline & \multirow[b]{2}{*}{ Vertical $(z)$} & $X, Y$ & $\begin{array}{l}\text { - Either the same or different simultaneously for both } x \text { - and } y \text {-QNs } \\
\text { - Different for the } z \text {-QN }\end{array}$ \\
\hline & & $Z, S$ & $\begin{array}{l}\text { - The same for both } x \text {-and } y \text {-QNs or the initial parity or the } x-\mathrm{QN} \text { is the same } \\
\text { than the final parity of the } y \text {-QN and vice versa } \\
\text { - Irrelevant for the } z-\mathrm{QN}\end{array}$ \\
\hline
\end{tabular}


and the tables of forbidden elements are modified. Thus, Table 8 is not applicable to these cases.

Note that, since the transitions $h h \rightarrow c b$ and $s o \rightarrow c b$ follow the same rules, they apply to the standard and the interchanged configuration as well.

\section{The absorption coefficient}

As in Ref. [16], the calculation of the matrix elements allows the calculation of the absorption coefficient. The equation is given by

$\alpha_{x, \text { line }}=2 \times \frac{2 \pi^{2} e^{2} E}{n c h \varepsilon_{0}} \frac{|M x|^{2}}{4 a^{2}} F N_{l} \delta\left(E-E_{\text {line }}\right)$

where $e, n, h, c$, and $\varepsilon_{0}$ are the electron charge, the index of refraction, the Planck constant, the speed of the light in vacuo and the electric permittivity of the vacuum, respectively; $F$ is the fraction of surface occupied by QDs, $N_{l}$ is the density of QD layers per $\mathrm{cm}$ (see Fig. 1), $E$ (independent variable; in the context of symmetry it is also the identity) is the energy and $E_{\text {line }}$ is the difference of energies between the final and initial states of the transition. The first factor 2 accounts for the double spin degeneracy. Equivalent expressions hold for other polarizations. Notice that for vertical unpolarized light $\alpha_{\text {line }}=\left(\alpha_{x, \text { line }}+\alpha_{y, \text { line }}\right) / 2$. This coefficient has the shape of a Dirac delta but it is smoothed by non-homogeneities in a form that here, as in Ref. [16], is taken as a Gaussian of standard deviation $0.025 / \sqrt{ } 2 \mathrm{eV}$.

Fig. 6 shows the absorption coefficient of the transitions ending in the different $c b$ energy levels. They are obtained by adding all the line absorption coefficients starting in $h h$, $l h$ or so and ending at the corresponding $c b$ energy level. The absorption coefficients are symmetric with respect to the permutation of the $n_{x}$ and $n_{y}$ QNs; e.g., $211 h h$ in the figure (note $h h$ at the end means the transition from all the $h h$ states to $c b(2,1,1)$ ) and a similar curve is valid for the transition to $c b(1,2,1)$; thus in these cases the absorption coefficient is multiplied by 2 . All the VB confined states ( $h h$, Ih and so) are considered to be completely filled with electrons and all cb states are considered to be completely empty, with the exception of the $c b(1,1,1)$ state, which is considered to be half filled by doping; hence, the absorption coefficient for transitions to the $c b(1,1,1)$ state is multiplied by $1 / 2$. The black line in the plot represents the total absorption coefficient due to transitions form the $V B$ confined states to the $C B$ confined states falling within the GaAs band gap. It is formed by the sum of the absorption coefficients to the individual energy states.

The Internal Quantum Efficiency (IQE) was also calculated in Ref. [16] under the assumption that every absorption leads to the delivery of an electron to the external circuit. This assumption is reasonable because the experimental IQE is measured taking care that the photocurrent is saturated, using reverse bias if necessary. In this paper it is also calculated in the standard and interchanged configurations for several values of the rotation, $\alpha$. This is presented in Fig. 7.

The calculations for small $\alpha$ agree reasonably with the measured IQE, at least in the standard configuration. They agree better than those in the precedent [16], particularly better reproducing the increase of the absorption coefficient in the high energy part of the curve. This was one of the unsatisfactory model aspects mentioned in [16]. There is a slight blue shift of about $11 \mathrm{meV}$ in the IB peak because the transition $h h(1,1,1) \rightarrow c b(1,1,1)$ is forbidden (it was not in [16]); this can easily be tuned by changing
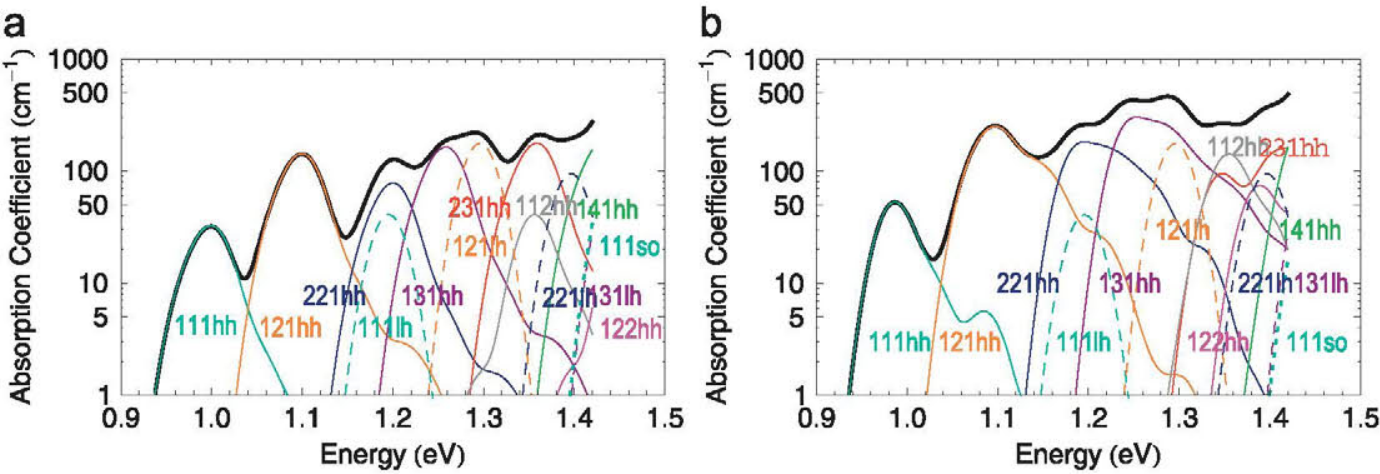

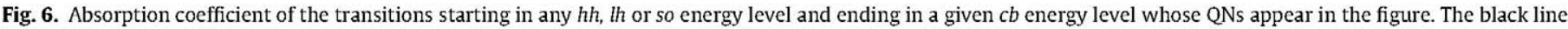
is the absorption coefficient for the transitions to all the $c b$ energy levels. Rotation parameter $\alpha=0.3$. (a) standard configuration; (b) interchanged configuration.

a

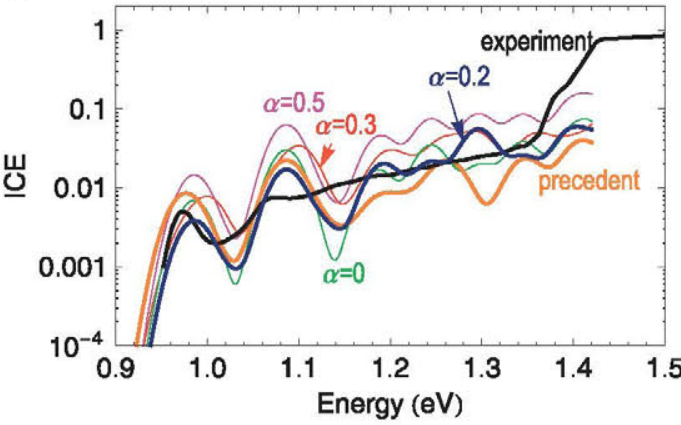

b

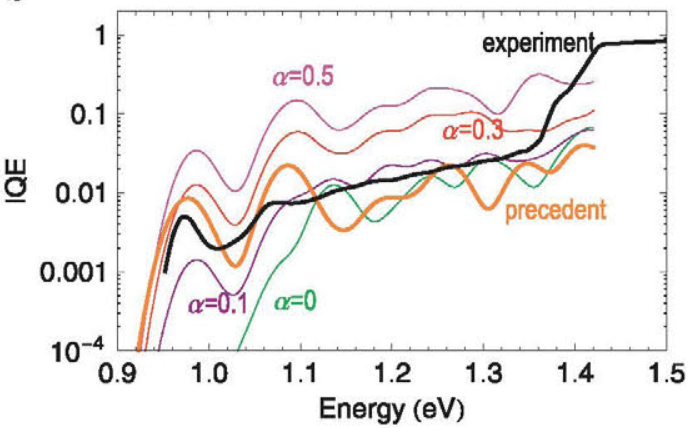

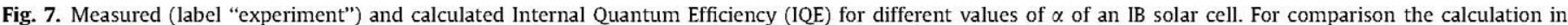
reference [16] (label "precedent") is also presented. (a) standard configuration; (b) interchanged configuration. 


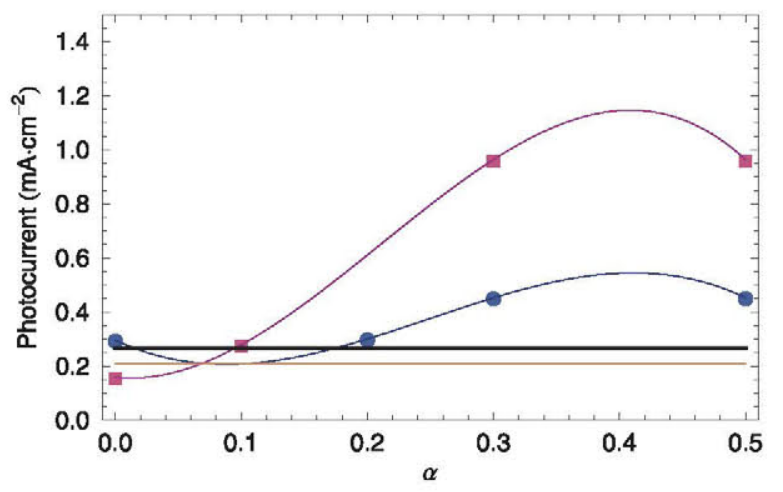

Fig. 8. Sub-bandgap photocurrent for calculated cases for several values of $\alpha$ for the standard configuration (blue dots) and for the interchanged configuration (red squares). The black horizontal line represents the experimental sub bandgap photo-current and the orange line the photo-current calculated in reference [14]. (For interpretation of the references to color in this figure legend, the reader is reffered to the web version of this article.)

slightly the CB offset. In all cases, the calculated curves are wavier than the measured one, but ideally the IQE is formed of a collection of Dirac- $\delta$ shaped lines resulting from Eq. (17). The continuous aspects result from non-homogeneities smoothed by adopting a Gaussian for the Dirac- $\delta$. The standard deviation adopted should not be much larger because then the leading edge of the IQE would be more inclined. However, we cannot assure that the same standard deviation must be used for all the absorption lines. In any case the IQE integration, from the onset of the absorption curve to $1.36 \mathrm{eV}$ (when the absorption by wetting layer formed in the manufacturing process appears) weighted with a solar spectrum (blackbody at $5762 \mathrm{~K}$, power of $1350 \mathrm{~W} \cdot \mathrm{m}^{-2}$, very close to the extraterrestrial solar radiation) gives $0.266 \mathrm{~mA} \cdot \mathrm{cm}^{-2}$ for the measured cell and similar values for several cases calculated, as presented in Fig. 8.

It can be seen that the integral agreement is very good in the standard configuration up to $\alpha \leq 0.2$ and in the interchanged configuration for $\alpha=0.1$. However, in the latter option, the direct transitions to the $c b(1,1,1)$ state are too small.

All taken into account, we conclude that the best fitting is produced with the standard configuration for $\alpha=0.2$. This case gives a direct absorption by the $c b(1,1,1)$ state (the IB) slightly below the experimental one, but we know today that the filling factor of this state is not 0.5 , as used in our calculations to compare with past calculations, but 0.36 , leaving 0.64 of the states empty. This would lead the calculated absorption coefficient to underestimate the measured value.

\section{Conclusions}

The experimental k.p Hamiltonian (EKPH) for calculating the absorption of QD intermediate band solar cells is based on two basic assumptions. The first is that the dispersion $E(\boldsymbol{k})$ curves of the different bands, which are the eigenvalues, are based on their measured effective masses. This allows calculation of the energy spectrum in the presence of QDs based on simple Schrodinger equations with effective masses. The rest of the assumptions in the paper - the square potential wells, the box shaped form of the $\mathrm{QD}$, and the separation-of-variables methods - are contingent, although they are often used with reasonable results.

The calculation of the matrix elements of the optical transitions requires the second basic assumption that we express succinctly now. It requires the calculation of the envelopes associated to each involute. For this we use a four band $\mathrm{k} \cdot \mathrm{p}$ approximation and we derive the matrix $\{T\}$ - that transforms the diagonal Hamiltonian of the experimental eigenvalues into the Hamiltonian corresponding to the basis system comprising the involutes - from the one where the spin-orbit and strain interactions are neglected (the use of the experimental eigenvalues assures that these effects are taken into account empirically). Since this simplified Hamiltonian has a doubly degenerate eigenvalue, the corresponding eigenvectors (that forms $\{T\}$ ) can be chosen amongst the infinite possibilities within the resulting vector space. The first choice made is a pair of orthonormal eigenvectors that transform in the simplest way under the symmetry group of the Hamiltonian - each of their elements follows an irreducible representation of $\mathrm{D}_{4 \mathrm{~h}}$. The remaining possibilities are then sampled by rotating this pair by a rotation parameter $\alpha$ - where the rotation axis is defined so as to preserve the symmetry between the $X$ - and $Y$-envolvents - and by swapping which eigenvector corresponds to the $h h$ and which to the so band. Absorption spectra are presented for all possible cases and the role of $\alpha$ is discussed. The agreement of the calculated absorption spectrum using the here presented model is found to be better than that using the previous model, presented in Ref. [16], in which a detailed study of symmetry was absent. The best agreement with experimental results is found for small $\alpha$.

It is confirmed that the weakness of the absorption is mostly due to the fact that the $\mathrm{CB}$ confined states have a small projection onto the VB $X$ - and $Y$-involutes and that the VB confined states have a small projection onto the CB S-involute. How to increase the overlap between $\mathrm{VB}$ and $\mathrm{CB}$ states by design of the $\mathrm{QD}$ structure remains an open question.

In addition to this, a study of the role of the symmetry is undertaken in the framework of the theory representations in order to extract the forbidden optical transitions and reduce the calculation burden. This is not trivial; it is found that the determination of the forbidden transitions requires the Cartesian product of five representations of the QD symmetry group $\left(D_{4 h}\right)$, some of which were not irreducible but the direct sum of two irreducible representations. All the relevant representations have been determined. Easy recipes are given to determine the forbidden states of the different transitions.

From a physical point of view, we think that a rather clear picture of the sub-bandgap absorption process is now available. The IB or $c b(1,1,1)$ confined state is partially filled of electrons due to $n$-doping of the $\mathrm{CB}$ and receives electrons pumped by photons from the $h h$ states that form a deep VB queue inside the band gap (see Fig. 1) and also form the $l \mathrm{~h}$ and so bands (the latter with negligible influence because it requires overly energetic photons), but this is only a small part of the sub-bandgap photocurrent. Many of the photons are absorbed by transitions to the remaining $c b$ confined states, and in particular by $c b(1,2,1), c b(2,2,1)$ and $c b(1,3,1)$ (see Fig. 1 and Fig. 6) originated in the $h h$ and $l h$ bands. Numerous transitions take place among these $c b$ states [13] associated to absorptions and emissions of low energy photons (of about $100 \mathrm{meV}$ ) taken from the huge reservoir of thermal photons within the semiconductor and to a lesser extent from photons coming from outside. Finally, they end in confined states of larger QNs embedded in the $\mathrm{CB}$ and from there they pass to the external circuit. For instance, the easier path for the IB state $(c b(1,1,1))$ to the $\mathrm{CB}$ is $[13] c b(1,1,1) \rightarrow c b(1,2,1) \rightarrow c b(1,3,1)$.

Finally, there is another component of the sub bandgap absorption that has not yet been studied: the transitions from the extended states in the VB to the localized cb states within the bandgap. An analogous study was made in [14] of the transitions between the confined and extended CB states. It was found that, although the overlap between confined and extended states is small, the high density of extended states leads to a significant absorption coefficient. It would be expected that the situation is similar for transitions from extended states in the VB to confined 
states in the $\mathrm{CB}$. These transitions are important since they contribute to the sub-bandgap photocurrent. In our example cell, their onset will be for photons with energy greater than $1.16 \mathrm{eV}$, although they will only be significant for photons above $1.26 \mathrm{eV}$ (when the VB density of states becomes significant). Absorption involving a continuum of extended states is generally smoother than that associated only to confined states; hence the absence of this absorption could explain why the experimental IQE is smother tan that calculated in this and previous models.

\section{Acknowledgment}

This work has been supported by the European Commission through the project NGCPV (Grant 283798) for EU-Japan Cooperation, by the Spanish Ministry of Science and Innovation through the project DENQUIBAND (Grant PLE2009-0045), for Spain-Japan Cooperation and NANOGEFES: (Grant ENE2009-14481-C02-01).

\section{Appendix. Selected symbols and acronyms}

$\begin{array}{ll}\mathrm{CB} & \text { conduction band } \\ c b & \text { conduction band state } \\ \mathrm{D}_{4 \mathrm{~h}} & \text { symmetry group of the QDs } \\ \mathrm{EKPH} & \text { empirical k.p Hamiltonian } \\ H & \text { Hamiltonian } \\ H_{d} & \text { diagonalized Hamiltonian } \\ H_{d}^{\prime} & \text { diagonalized EKPH } \\ h h & \text { heavy hole state } \\ \mathrm{IB} & \text { intermediate band } \\ \mathrm{IBSC} & \text { intermediate band solar cell } \\ \mathrm{IQE} & \text { internal quantum efficiency } \\ l h & \text { light hole state } \\ \mathrm{MBE} & \text { molecular beam epitaxy } \\ \mathrm{QD} & \text { quantum dot } \\ S & \text { involute wavefunction } \\ s o & \text { split off state } \\ T_{\alpha} & \text { transformation matrix } \\ \mathrm{VB} & \text { valence band } \\ X & \text { involute wavefunction } \\ Y & \text { involute wavefunction } \\ Z & \text { involute wavefunction } \\ \chi & \text { character of a representation } \\ \Phi & \text { diagonalized eigenfunction } \\ I & \text { point } k=0 \text { in the reciprocal space } \\ \Xi & \text { wavefunction } \\ \Psi & \text { envolvent wavefunction } \\ & \end{array}$

\section{References}

[1] A. Martí, L. Cuadra, and A. Luque, Quantum dot intermediate band solar cell, Proc. 28th IEEE Photovoltaics Specialists Conference, IEEE, New York, 2000, pp. $940-943$.
[2] A. Martî, L. Cuadra, A. Luque, Partial filling of a quantum dot intermediate band for solar cells, IEEE Transactions on Electron Devices 48 (2001) 2394-2399.

[3] A. Luque, A. Martí, Increasing the efficiency of ideal solar cells by photon induced transitions at intermediate levels, Physical Review Letters 78 (1997) 5014-5017.

[4] A. Luque, A. Martí, A metallic intermediate band high efficiency solar cell, Progress in Photovoltaics: Research and Applications 9 (2001) 73-86.

[5] W. Shockley, H.J. Queisser, Detailed balance limit of efficiency of p-n junction solar cells, Joumal of Applied Physics 32 (1961) 510-519.

[6] A. Luque, A. Martí, C. Stanley, N. López, L. Cuadra, D. Zhou, A. Mc-Kee, General equivalent circuit for intermediate band devices: potentials, currents and electroluminescence, Journal of Applied Physics 96 (2004) 903-909.

[7] S.M. Hubbard, C.D. Cress, C.G. Bailey, R.P. Raffaelle, S.G. Bailey, D.M. Wilt, Effect of strain compensation on quantum dot enhanced GaAs solar cells, Applied Physics Letters 92 (2008) 123512.

[8] R. Oshima, A. Takata, Y. Okada, Strain-compensated InAs/GaNAs quantum dots for use in high-efficiency solar cells, Applied Physics Letters 93 (2008) 083111.

[9] Y. Okada, T. Morioka, K. Yoshida, R. Oshima, Y. Shoji, T. Inoue, T. Kita, Increase in photocurrent by optical transitions via intermediate quantum states in direct-doped InAs/GaNAs strain-compensated quantum dot solar cell, Journal of Applied Physics 109 (2011) 5.

[10] S.A. Blokhin, A.V. Sakharov, A.M. Nadtochy, A.S. Pauysov, M.V. Maximov, N.N. Ledentsov, A.R. Kovsh, S.S. Mikhrin, V.M. Lantratov, S.A. Mintairov, N.A. Kaluzhniy, M.Z. Shvarts, AlGaAs/GaAs Photovoltaic Cells with an Array of InGaAs QDs, Semiconductors 43 (2009) 514-518.

[11] C.E. Valdivia, S. Chow, S. Fafard, O. Theriault, M. Yandt, J.F. Wheeldon, A.J SpringThorpe, B. Rioux, D. McMeekin, D. Masson, B. Riel, V. Aimez, R. Ares, J. Cook, T.J. Hall, F. Shepherd, K. Hinzerand leee, , Measurement of high efficiency $1 \mathrm{~cm}^{2} \mathrm{AlGaInP} / \mathrm{InGaSs} / \mathrm{Ge}$ solar cells with embedded InAs quantum dots at up to 1000 Suns continuous concentration, 35th IEEE Photovoltaic Specialists Conference, IEEE, New York, pp. 1253-1258.

[12] A. Luque, A. Marti, E. Antolin, P. Garcia-Linares, Intraband absorption for normal illumination in quantum dot intermediate band solar cells, Solar Energy Materials and Solar Cells 94 (2010) 2032-2035.

[13] A. Luque, A. Marti, E. Antolin, P.G. Linares, I. Tobias, I. Ramiro, Radiative thermal escape in intermediate band solar cells, AIP Advances 1 (2011) 022125 .

[14] A. Luque, A. Marti, A. Mellor, D.F. Marron, I. Tobias, E. Antolín, Absorption coefficient for the intraband transitions in quantum dot materials, Progress in Photovoltaics (2012)http://dx.doi.org/10.1002/pip.1250.

[15] S. Datta, Ouantum Phenomena, Addison Wesley, Reading (Mass), 1989

[16] A. Luque, A. Marti, E. Antolín, P.G. Linares, I. Tobías, I. Ramiro, E. Hernandez, New Hamiltonian for a better understanding of the quantum dot intermediate band solar cells, Solar Energy Materials and Solar Cells 95 (2011) 2095-2101.

[17] L.C.L.Y. Voon, M. Willatzen, The k.p Method, Springer, Berlin, 2009

[18] A. Messiah, Mécanique Quantique, Dunod, Paris, 1960.

[19] J.-M. Iniguez-Almech, Mecanica Cuantica, Academia de Ciencias de Zaragoza, 1949.

[20] W.P. Atkins, M.S. Child, C.S.G. Phillips, Tables for Group Theory, Oxford University Press, Oxford, 2006.

[21] E. Kaxiras, Atomic and Electronic Structure of Solids, Cambridge University Press, New York, 2003.

[22] E. Antolín, A. Marti, C.D. Farmer, P.G. Linares, E. Hernândez, A.M. Sánchez, T. Ben, S.I. Molina, C.R. Stanley, A. Luque, Reducing carrier escape in the InAs/ GaAs quantum dot intermediate band solar cell, Journal of Applied Physics 108 (2010) 064513.

[23] V. Popescu, G. Bester, M.C. Hanna, A.G. Norman, A. Zunger, Theoretical and experimental examination of the intermediate-band concept for strainbalanced (In,Ga)As/Ga(As,P) quantum dot solar cells, Physical Review B 78 (2008) 205321.

[24] P. Harrison, Quantum Wells Wires and Dots, John Wiley and Sons, New York, 2000 . 